\title{
Improving P300 Speller performance by means of optimization and machine learning
}

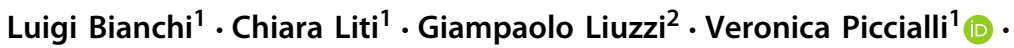 \\ Cecilia Salvatore ${ }^{1}$
}

Accepted: 24 December 2020 / Published online: 20 January 2021

(c) The Author(s) 2021

\begin{abstract}
Brain-Computer Interfaces (BCIs) are systems allowing people to interact with the environment bypassing the natural neuromuscular and hormonal outputs of the peripheral nervous system (PNS). These interfaces record a user's brain activity and translate it into control commands for external devices, thus providing the PNS with additional artificial outputs. In this framework, the BCIs based on the P300 Event-Related Potentials (ERP), which represent the electrical responses recorded from the brain after specific events or stimuli, have proven to be particularly successful and robust. The presence or the absence of a P300 evoked potential within the EEG features is determined through a classification algorithm. Linear classifiers such as stepwise linear discriminant analysis and support vector machine (SVM) are the most used discriminant algorithms for ERPs' classification. Due to the low signal-tonoise ratio of the EEG signals, multiple stimulation sequences (a.k.a. iterations) are carried out and then averaged before the signals being classified. However, while augmenting the number of iterations improves the Signal-to-Noise Ratio, it also slows down the process. In the early studies, the number of iterations was fixed (no stopping environment), but recently several early stopping strategies have been proposed in the literature to dynamically interrupt the stimulation sequence when a certain criterion is met in order to enhance the communication rate. In this work, we explore how to improve the classification performances in P300 based BCIs by combining optimization and machine learning. First, we propose a new decision function that aims at improving classification performances in terms of accuracy and Information Transfer Rate both in a no stopping and early stopping environment. Then, we propose a new SVM training problem that aims to facilitate the target-detection process. Our approach proves to be effective on several publicly available datasets.
\end{abstract}

Keywords Brain computer interface · MILP mixed integer linear programming · P300 Speller · Support vector machine

\section{Introduction}

A Brain-Computer Interface (BCI) is a system that records a user's brain activity and allows him to interact with the environment by exploiting both signal processing and machine

Extended author information available on the last page of the article 
learning algorithms. In most cases, the recorded signals are noisy, so that filtering or averaging techniques are used to improve the signal-to-noise ratio (SNR). The information embedded in signals that are relevant to characterize the user's mental states are then selected during a feature extraction procedure before being classified and translated into artificial outputs-i.e. into control commands for an output device such as a pointer, a keyboard or a robotic arm (Lotte 2014; Lotte et al. 2018; Quitadamo et al. 2008; Wolpaw and Wolpaw 2012; McCane et al. 2015). BCIs use either electrical, magnetic and metabolic signals (Wolpaw and Wolpaw 2012) recorded with methods such as electroencephalography (EEG), electrocorticography (ECoG), magnetoencephalography (MEG), functional Near Infra-Red Spectroscopy (fNIRS) and functional Magnetic Resonance Imaging (fMRI). In particular, EEG represents one of the most used methods since they are non invasive and inexpensive; for this reason they have been used for a wide variety of tasks (Chaovalitwongse et al. 2006; Khojandi et al. 2019).

In this framework, BCIs based on event-related potentials (ERPs) have proven to be particularly successful and robust (Schreuder et al. 2013). ERPs represent the electrical responses recorded from the brain through EEG techniques after specific events or stimuli. The ERPs are embedded within the general EEG activity (Sur and Sinha 2009), and are timelocked to the processing of a specific stimulus. As their amplitude is lower that the one of the ongoing EEG activity, averaging techniques are employed to increase the SNR: in principle, averaging background noise which is not correlated to an event, such as the ongoing EEG activity, tends to reduce its contribution to a small offset, which can be easily filtered out, while the evoked responses, supposed to be the same after each stimulus, are left unmodified. An ERP-based BCI attempts to detect ERP components to infer the stimulus that the user intended to choose-i.e. the stimulus eliciting the ERP components (Treder and Blankertz 2010; Shahriari et al. 2019).

In 1988, the P300 ERP was first used by Farwell and Donchin within a BCI system (Farwell and Donchin 1988). Their P300 Speller consists of 36 alpha-numeric characters arranged within the rows and columns of a $6 \times 6$ matrix. The user's task is to focus the attention on a specific character-i.e. on one of the cells of the matrix. Each of the 6 rows and 6 columns then flashes for few tenths of milliseconds in a random sequence. A sequence of 12 different flashes - the 6 rows and 6 columns - is called an iteration. It constitutes the basis of an oddball paradigm in which two classes of stimuli, namely the target (or rare) and the non target (or frequent) which occur with different probabilities (0.166 and 0.833 in this case), elicit two different brain responses. In particular, the target (rare) stimuli should elicit the P300 response, which should not be evoked after a non target (frequent) stimuli. In our case the row and the column containing the attended character represent the target stimuli, while the other ten are the non-target ones. Brain responses to the target and non-target stimuli are distinguished using a classification algorithm. The correct identification of the target row and column allows the desired character's selection, which is located at their intersection (Krusienski et al. 2006, 2008; Sellers et al. 2006).

Later on in the literature, different variations of the original P300 paradigm have been developed in order to improve the speller framework. For instance, in Schaeff et al. (2012), Schreuder et al. (2011), Treder et al. (2011) the authors proposed gaze-independent spellers, i.e. communication systems that can be used by subjects who have impairment at moving their eyes. In all speller paradigms, given a sentence/run to copy-spell, the EEG data are organized in terms of trials, iterations, and sub-trials. A single character selection step is here referred to as a trial. Each trial consists of several iterations/stimulation sequences, during which all the stimuli are intensified once in a pseudo-random order. A single stimulus intensification is here referred to as a sub-trial. The trials' selection process usually involves one or two levels. In the former case, symbols are typically presented successively 
thus involving a single selection step. In the latter, the user has to select a group of symbols at the first level and then the target symbol at the second level.

To use a BCI, two phases namely training/calibration and test/online are typically required. During the calibration phase, the user is instructed to focus his/her attention on a specific character (copy task) for which correct labels are then a priori known. The acquired EEG signals are then preprocessed by filtering. A subset of EEG features is extracted to represent the signal in a compact form. The obtained EEG patterns are recognized using a classification algorithm, which is trained on the subset of identified features to determine the presence or the absence of a P300 evoked potential. In the online phase, new EEG patterns are classified using the trained model before being translated into a command for an application. As described above, in ERP-based BCIs, to perform a single selection step, multiple iterations are carried out to improve the SNR. Since each iteration takes about 3 seconds to be completed, this strategy increases the time needed to detect brain signals thus affecting down the communication rate. To overcome this drawback, different early stopping (ES) or Dynamic Stopping methods have been introduced, where after a calibration phase, a suitable termination criterion is established to be tested online when the number of iteration is sufficient to ensure a reliable classification. In this work, we explore how to improve the classification performance by combining optimization and machine learning both in the classical setting with a fixed number of repetitions and in the early stopping setting.

\subsection{Literature review}

As mentioned above, the presence or the absence of a P300 evoked potential within the EEG features is determined using a classification algorithm (Krusienski et al. 2006).

Formally, the detection of brain responses to the target and non-target stimuli can be translated into a binary classification problem. Let $T S$ be the training set defined as:

$$
\begin{aligned}
T S & =\left\{\left(x_{i}, y_{i}\right): x_{i} \in \mathbb{R}^{n}, y_{i} \in\{-1,+1\}\right. \\
i & \left.:=(k, r, t, f) \quad \forall k=1 \ldots n_{k}, r=1 \ldots n_{r}, t \in T, f=1 \ldots n_{f}\right\}
\end{aligned}
$$

where $n_{k}$ denotes the total number of trials in the training phase and $n_{r}$ denotes the number of iterations for each trial; the number of flashes $n_{f}$ and the set of levels $T$ together denote the set of possible stimuli that compose the stimulation sequence (i.e. $n_{f}=6$ and $T=\{$ row, column $\}$ for P300 Speller's paradigm or $T=\{$ outer, inner $\}$ for two-levels paradigms).

During the calibration phase, a classification algorithm is trained over $T S$ to learn the discriminant function $f$ such that

$$
f(x)=y,
$$

and this function is used in the online phase to spell words or sentences. In the BCI literature, several algorithms have been proposed for addressing this classification problem (Lotte et al. 2018). In particular, linear classifiers such as stepwise linear discriminant analysis (SWLDA) (Draper and Smith 1998), and support vector machine (SVM) (Friedman et al. 2001) are still the most used discriminant algorithms for ERPs' classification (Lotte et al. 2018). These methods classify the brain responses by means of a separating hyperplane (Krusienski et al. 2006). This discriminant function is built on the basis of the training data, and it is defined as:

$$
f(x)=w^{T} x+b,
$$


where $w$ is the vector containing the classification weights and $b$ is the bias term. Linear classifiers differ in the way they learn $w$ and $b$ (Krusienski et al. 2006). In (3), the right-hand side is called decision value. Its absolute value is proportional to the distance of the sample points $x$ from the separating hyperplane.

In a standard binary classification problem, for each instance the class label is assigned based on the sign of the relative decision value. However, in a classical P300 Speller (Farwell and Donchin 1988), based on the assumption that a P300 is elicited for one of the six row/column stimuli and finding that the P300 response is invariant to row/column stimulation, the target class is assigned to the stimuli matching the maximum decision values for both the rows and the columns (Krusienski et al. 2006). In general, recalling the definition of $T$ and $n_{f}$ given in (1), we can identify the target stimulus for trial $k \in\left\{1 \ldots n_{k}\right\}$ and iteration $r \in\left\{1 \ldots n_{r}\right\}$ as:

$$
\operatorname{predicted~stimulus~}_{(k, r, t)}=\underset{f=1 \ldots n_{f}}{\operatorname{argmax}}\left[w^{T} x_{(k, r, t, f)}+b\right] \quad \forall t \in T
$$

The predicted character for trial $k \in\left\{1 \ldots n_{k}\right\}$ and iteration $r \in\left\{1 \ldots n_{r}\right\}$ is then identified by combining the predicted target stimuli found $\forall t \in T$ (i.e a row target and a column target for the standard P300 paradigm).

As mentioned in Sect. 1, for each character, data recorded from multiple iterations have to be integrated to improve the SNR. To the best of our knowledge there exist two main different iteration-averaging strategies in the literature: (i) ERP avg: for each character brains responses to target and non target stimuli are averaged across the iterations before being classified, and (ii) DV avg: for each character the decision values of each target and nontarget stimulus are averaged across the iterations before assigning the target class. Recently in (Bianchi et al. 2019), a new classification function namely score-based function (SBF) has been introduced for integrating brain responses recorded from multiple iterations. For each character, the SBF exploits a set of heuristically-determined scores to weight each stimulus according to its decision value. For each stimulus, the assigned scores are summed up iteration by iteration. The target class (one for the row and one for the column) is assigned to the stimulus having the highest total score after the last available iteration. The SBF has been introduced for developing an early stopping method (ESM)-i.e. an automatic method that interrupts the stimulation at any point in a trial when a certain criterion, based on the ongoing classification results, is satisfied (see for instance Lenhardt et al. 2008; Zhang et al. Jun 2008; Liu et al. 2010; Höhne et al. 2010; Schreuder et al. 2011; Jin et al. 2011; Throckmorton et al. 2013; Mainsah et al. 2014; Jiang et al. 2018; Vo et al. 2017, 2018; Schreuder et al. 2013; Kha et al. 2017; Gu et al. 2019; Huang et al. 2020). The proposed ESM based on the SBF outperformed the current state-of-the-art early stopping methods proposed in Schreuder et al. (2013). Note that the SBF is quasi-opposite to the approach proposed in Kha et al. (2017) where a score is assigned to an SVM based classifier and then an Early Stopping is defined using the cumulative scores of the classifiers. In Huang et al. (2020), an Early Stopping technique is used to improve the accuracy of the P300 speller while it is performing other tasks. Thus the proposed approach allows adapting the classification accuracy to the subject's attention level in real-time. In this paper, we follow the same line of research of Bianchi et al. (2019), by making some further steps to include the information on the protocol into the classification phase. Indeed, the novelty of our approach consists of three points:

1. determine the optimal scores for each participant by solving an optimization problem on her/his training data; 
2. solve a modified version of the optimization problem in order to implement an efficient early stopping method;

3. include the information on the decision function (the target is the stimulus having maximum decision value) into the training problem

The great advantage of our method is that the calibration phase (different for each participant) becomes completely automatic and does not need any cross validation phase or manual parameters tuning.

The paper is structured as follows: in Sect. 2, we introduce our new decision function, defining the optimization problems to be solved both in the no stopping and early stopping scenario. In Sect. 3, we introduce a new training problem that keeps into account explicitly the target assignment in BCI, and in Sect. 4 we derive its Wolfe dual. In Sect. 6 we report the behavior of our new approaches on several datasets and finally we draw some conclusions in Sect. 7. In "Appendix 1" we quickly describe the algorithm for solving the dual of our new training problem, while in Appendix 2 we report the detailed numerical results for all the datasets.

\section{An optimized score based decision function (OSBF)}

In Bianchi et al. (2019), a set of heuristically-determined scores has been used to weight and combine the decision values of multiple iterations within an early stopping setting. In this work, we decided to modify the approach by using a set of scores automatically determined by solving a mixed integer linear programming (MILP) problem for each participant. Each stimulus receives a weight according to its decision value: five zones are defined, and each zone gets a different score $a, b, c, d, e$. In particular, the scores are related to the confidence in the classification of the given stimulus as target: the score $a$ is assigned to the stimulus that is most likely to be the target, whereas the stimuli that are highly unlikely to be the target get score $e$. All the stimuli in the middle get decreasing scores according to the distribution of the decision values.

The zones are identified by considering the decision values of all iterations for all stimuli in the training set and computing the corresponding quartiles Q1, Q2 and Q3. The idea is to produce scores that reflect the distribution of the data.

Figure 1 shows how the scores are assigned depending on the distribution of the quartiles of the decision values in a simple 2-dimensional example. The maximum score $a$ is assigned only if the confidence in the current classification is extremely high: i.e. if the decision value is positive and higher than all the other decision values of the current iteration.

Note that, given the separating hyperplane, the score assignment for each stimulus of each character is known: so, it is possible to build the following binary vectors that represent in a compact form the score vector assignment $z$ for each stimulus of each character:

$$
z_{s}^{k, r, t, f}=\left\{\begin{array}{l}
1 \text { if stimulus } f \text { of level } t \text { gets score } s \text { at iteration } r \text { for char } k \\
0 \text { otherwise }
\end{array}\right.
$$

where $f=1 \ldots n_{f}$ and $t \in T$ identify the stimulus, $k=1 \ldots n_{k}$ identifies the character, $r=1 \ldots n_{r}$ identifies the iteration and, finally, $s=a, \ldots, e$ identifies the score. The score assignments depends on the primary aim of the BCI:

(i) if the main focus is the accuracy, the idea is to use all the available iterations for spelling a character (no stopping protocol), also in the online phase. 


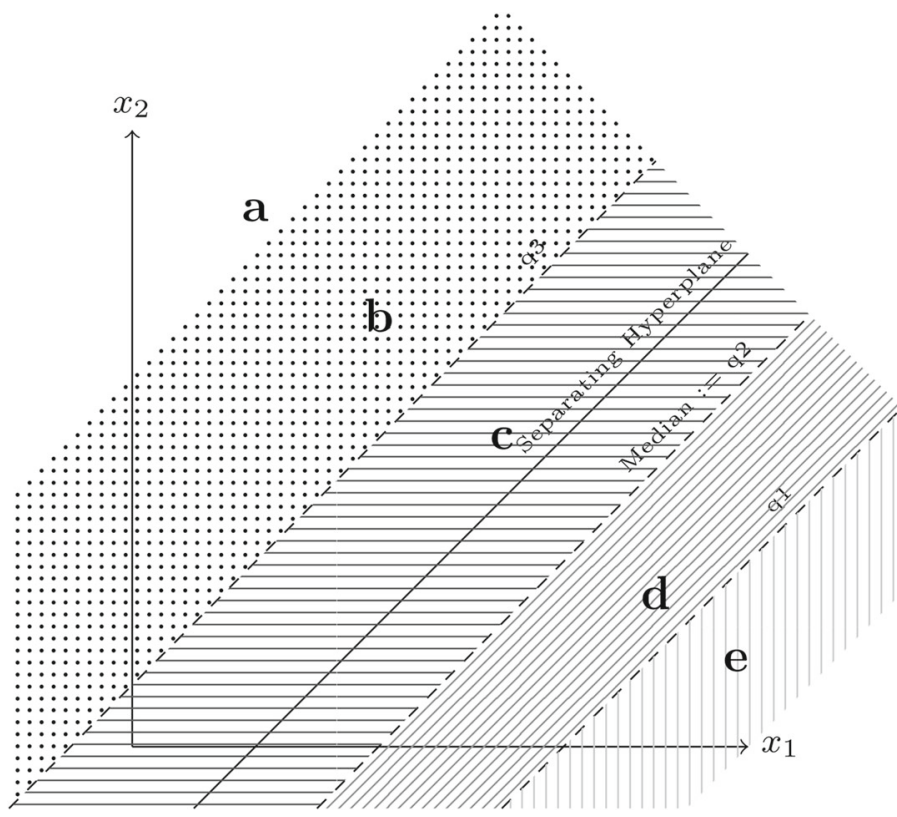

Fig. 1 Graphical representation of the score distribution, reflecting the displacement of the points w.r.t the distribution of the decision values. The different areas represent the confidence of the classification w.r.t the target class. Please note that this is a simplified example where samples are represented as 2-dimensional data points with features $x_{1}$ and $x_{2}$

(ii) if the idea is to try and speed up the communication, then the performance to be maximized is the transmission rate, trying to reduce the number of iterations needed to spell a character in the online phase (early stopping).

In the next two subsections, we describe the Mixed Integer Linear Programming (MILP) Problems we define in order to find the scores in the two different settings.

\subsection{No stopping OSBF}

First, we propose a strategy to choose the scores when all the iterations are exploited and the primary focus is to increase the classification accuracy. In this setting, we aim at reliability of the classification and we do so by imposing the following constraints:

1. at the last iteration, we require, if possible, that the score obtained by the target stimulus is larger (with some margin if possible, that implies robustness of the classification) than the score of any non target stimulus. This means that we ask not to fail in the classification after the last available iteration; if this is not possible, a suitable binary variable representing the failure on that stimulus is set to one;

2. to make the classification more robust on the test set, we require that in as many iterations as possible, the score of the target is larger than the one of the non target stimuli;

3. as an objective, we try and maximize the accuracy on the training set, and the number of iteration where the classification is robust.

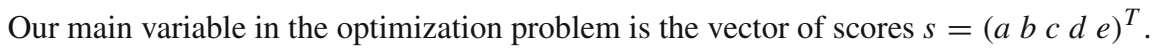


Table 1 Description of the set of indexes, parameters and variables used in the no stopping and early stopping OSBF

\begin{tabular}{|c|c|c|}
\hline \multicolumn{2}{|l|}{ Set } & Description \\
\hline \multicolumn{3}{|c|}{ Set of indexes } \\
\hline \multicolumn{2}{|l|}{$\mathrm{K}$} & Set of trials in the offline phase \\
\hline \multicolumn{2}{|l|}{$\mathrm{R}$} & Set of iterations that compose each trial \\
\hline \multicolumn{2}{|l|}{$\mathrm{T}$} & Set of levels of the paradigm \\
\hline \multicolumn{2}{|l|}{$\mathrm{F}$} & Set of stimuli in the stimulation sequence \\
\hline Name & Set of Indexes & Description \\
\hline \multicolumn{3}{|c|}{ Parameters } \\
\hline $\mathrm{z}$ & $\mathrm{K}, \mathrm{R}, \mathrm{T}, \mathrm{F}$ & $\begin{array}{l}\text { Binary vector of } 5 \text { components which represents the zones } \\
\text { partition. The } \mathrm{i} \text {-th component of } z^{k, r, t, f} \text { is set to } 1 \text { if and only } \\
\text { if stimuli }(\mathrm{k}, \mathrm{r}, \mathrm{t}, \mathrm{f}) \text { is assigned to the } \mathrm{i} \text {-th confidence zone }\end{array}$ \\
\hline Name & Set of Indexes & Description \\
\hline \multicolumn{3}{|c|}{ Variables } \\
\hline $\mathrm{x}$ & $\mathrm{K}, \mathrm{R}, \mathrm{T}$ & $\begin{array}{l}\text { Binary variable which equals } 1 \text { if the target stimuli is correctly } \\
\text { detected at current iteration in no stopping OSBF. For early } \\
\text { stopping OSBF, this variable equals } 1 \text { if the early stopping } \\
\text { condition is verified for the first time on the target at the } \\
\text { current iteration, and it is not satisfied by any non target } \\
\text { stimulus earlier }\end{array}$ \\
\hline err & \multirow[t]{3}{*}{$\mathrm{K}, \mathrm{T}$} & $\begin{array}{l}\text { Binary variable which equals } 1 \text { if the target stimuli was not } \\
\text { correctly detected at the last iteration possible }\end{array}$ \\
\hline s & & Score vector $s=\{a, b, c, d, e\}$ \\
\hline$\Delta$ & & Reliability Threshold \\
\hline
\end{tabular}

We add an auxiliary variable to try and impose some distance between the score of the target stimulus and the scores of the non target stimuli that we call $\Delta$, and that represents a measure of reliability of the classification. Further, we add some binary variables:

$-x_{t}^{k, r}$ : binary variable that is equal to 1 if the target of character $k$ for level $t$ has a score at iteration $r$ that is larger than the score of any non target stimulus plus $\Delta$

$-e r r_{t}^{k}$ : binary variable that is equal to 1 if the target is not correctly classified for character $k$ at level $t$, i.e. if at the last iteration the target score is lower or equal to the score of some non target stimulus

The MILP problem to be solved is then the following:

$$
\begin{aligned}
\max & \sum_{t \in T}\left(\left(1-\frac{\sum_{k=1}^{n_{k}} e r r_{t}^{k}}{n_{k}}\right)+\frac{1}{n_{k} n_{r}} \sum_{k=1}^{n_{k}} \sum_{r=1}^{n_{r}} x_{t}^{k, r}\right) \\
& s_{1} \leq u \\
& s_{j+1} \leq s_{j}-1 \forall j=1, \ldots, 4 \\
& s_{3} \geq 0 \\
& s_{5} \geq l \\
& \Delta \geq s_{1}-s_{5}+1
\end{aligned}
$$




$$
\begin{aligned}
& 1-\operatorname{err}_{t}^{k} \leq x_{t}^{k, n r} \forall k \in\left\{1 \ldots n_{k}\right\}, \forall t \in T \\
& \sum_{r=1}^{\bar{r}} s^{T} z^{k, r, t, f}+\Delta \leq \sum_{r=1}^{\bar{r}} s^{T} z^{k, r, t, \operatorname{trg}(k, t)} \\
& \quad+M\left(1-x_{t}^{k, \bar{r}}\right) \quad \forall k, \bar{r}, t, f: f \neq \operatorname{trg}(k, t) \\
& \operatorname{err}_{t}^{k}, x_{t}^{k, r} \in\{0,1\} \quad \forall k \forall t \forall r
\end{aligned}
$$

where $l$ and $u$ are chosen bounds on the possible values of the scores, and $M$ is large enough to make the constraints trivially satisfied when the corresponding binary variable $x_{t}^{k, r}$ is zero. The objective function, that has to be maximized, is composed by two terms: the percentage of success on the training set, and the average number of iterations where the classification is robust and reliable. We then have the following constraints:

(i) Constraints (6), (7) and (9) impose that the scores are bounded and that are ordered in decreasing order and differ of at least one; whereas constraint (8) imposes that the first three scores are nonnegative

(ii) constraint (10) imposes a lower bound on the threshold to ensure reliability of the classification. Indeed this lower bound ensures that the threshold has a minimum value depending on the scores: in particular $s_{1}-s_{5}+1$ represents the maximum difference in score that can be assigned to different flashes in a single iteration. Therefore, even in the worst possible scenario, where two flashes get the same score, there must be at least one iteration where one gets the maximum score and the other the minimum score to break the parity.

(iii) constraints (11) impose that variable $e r r_{t}^{k}$ is 1 if and only if $x_{t}^{k, n_{r}}=0$, that is it represents an unreliable classification at the last iteration.

(iv) constraints (12) impose that if at iteration $\bar{r}$ the classification is reliable for the target $\operatorname{tr} g(k, t)$ of character $k$ at level $t$, then the corresponding binary variable $x_{t}^{k, r}$ is set to 1 .

\subsection{Early stopping OSBF}

Problem (5) can be modified in order to improve the system performance in terms of speed, implementing an automatic Early Stopping Method, similarly to Bianchi et al. (2019).

The idea is again to use the scores $s$ and the threshold $\Delta$ at each iteration of the test phase to verify an early stopping condition: during the test phase, the stimuli are ordered according to the sum of their scores and, if the difference in score between the first and second stimulus is greater than the threshold $\Delta$, the method classifies the target character and the remaining iterations are not performed.

In order to adapt problem (5) to the early stopping setting, we introduce some further constraints, and modify the meaning of some binary variables:

$$
\begin{aligned}
\max & \sum_{t \in T}\left(1-\frac{1}{n_{k}} \sum_{k=1}^{n_{k}} e r r_{t}^{k}-\frac{100 \times n_{f l}}{60} \frac{S O A}{n_{k}}\left(\sum_{k=1}^{n_{k}} \sum_{r=1}^{n_{r}} r x_{t}^{k, r}+n_{r} \sum_{k=1}^{n_{k}} e r r_{t}^{k}\right)\right) \\
& (6)-(10) \\
& 1-\operatorname{err}_{t}^{k} \leq \sum_{r=1}^{n_{r}} x_{t}^{k, r} \forall k \in\left\{1 \ldots n_{k}\right\}
\end{aligned}
$$




$$
\sum_{r=1}^{n_{r}} x_{t}^{k, r} \leq 1 \forall k \in\left\{1 \ldots n_{k}\right\}
$$

$$
\begin{aligned}
& \sum_{r=1}^{\tilde{r}} s^{T} z^{k, r, t, f} \geq \sum_{r=1}^{\tilde{r}} s^{T} z^{k, r, t, f}-\Delta+1 \\
& \quad-M\left(1-x_{t}^{k, \bar{r}}\right) \quad \forall k, \forall t, \forall \tilde{r}<\bar{r}, \forall f: f \neq \operatorname{trg}(k, t) \\
& \operatorname{err}_{t}^{k}, x_{t}^{k, r} \in\{0,1\} \quad \forall k \forall t \forall r
\end{aligned}
$$

In this case, the objective function keeps into account both the percentage of success (to be maximized) and the time needed for classification (to be minimized). Note that the second term (which represents the trial duration in minutes) was multiplied by a factor 100 to make the two terms of the objective function comparable. We then have some further constraints, since in this case we are interested in the first iteration where the following early stopping condition is met:

$$
\sum_{r=1}^{\bar{r}} s^{T} z^{k, r, t, f}+\Delta \leq \sum_{r=1}^{\bar{r}} s^{T} z^{k, r, t, \operatorname{trg}(k, t)} .
$$

In this model, we set the binary variables $x_{t}^{k, r}$ in such a way that it is 1 if and only if the early stopping condition (19) is verified for the first time on the target at iteration $r$, and it is not satisfied by any non target stimulus earlier. This is imposed by the combination of constraints (12), (16) and (17).

We stress that in both the no stopping and the early stopping scenarios, the MILP problem is solved using the training set data (the same used to build the hyperplane), whereas the score efficiency is evaluated on the test set.

\section{A new training problem}

As already pointed out in the introduction, in order to achieve a good classification accuracy it is fundamental to exploit the information that at each iteration there is exactly one target stimulus for each level, assigning then the target class to the stimulus having the maximum decision value. Our idea is to try and add this protocol knowledge already in the training problem.

Given the definition (1) of training set, the standard training problem to solve in order to find a separating hyperplane according to the SVM approach is the following (Piccialli and Sciandrone 2018):

$$
\begin{aligned}
& \min _{w \in \mathbb{R}^{n}, b \in \mathbb{R}} \frac{1}{2}\|w\|^{2}+C_{1} \sum_{i \in T S} \xi_{i} \\
& y_{i}\left(w^{T} x_{i}+b\right) \geq 1-\xi_{i} \quad \forall i \in T S \\
& \xi_{i} \geq 0 \quad \forall i \in T S
\end{aligned}
$$

In this work, we modify the training problem including the information that the target stimuli should receive the maximum decision value among all the other flashes. Let's denote by $\operatorname{tr} g_{i}$ the target stimulus for the stimulation sequence where the stimulus $i$ belongs: so, in 
particular, if $i=(k, r, t, f)$ we will have:

$$
\operatorname{trg}_{i}=\left(k, r, t, f^{\prime}\right) \in T S \quad \& \quad y_{t r g_{i}}=1
$$

Then, we want to impose:

$$
\begin{aligned}
& w^{T} x_{\left(k, r, t, f_{1}\right)}+b \geq w^{T} x_{\left(k, r, t, f_{2}\right)}+b \quad \forall k, r, t, f_{1}, f_{2}: \\
& y_{\left(k, r, t, f_{1}\right)}=1 \& \quad y_{\left(k, r, t, f_{2}\right)}=-1
\end{aligned}
$$

From now on, in order to simplify the notation, we will write constraints (20) in the following more compact form:

$$
w^{T} x_{\text {trg }}+b \geq w^{T} x_{i}+b \quad \forall i \in T S: y_{i} \neq 1
$$

and we add slack variables to avoid infeasibility, getting the following set of constraints:

$$
\begin{aligned}
& w^{T} x_{\text {trg }}-w^{T} x_{i} \geq 1-\eta_{i} \quad \forall i \in T S: y_{i} \neq 1 \\
& \eta_{i} \geq 0 \quad \forall i \in T S: y_{i} \neq 1
\end{aligned}
$$

Now we simply plug these constraints into the primal problem getting the new training problem based on the maximum decision function:

$$
\begin{aligned}
& \min _{w \in \mathbb{R}^{n}, b \in \mathbb{R}} \frac{1}{2}\|w\|^{2}+C_{1} \sum_{i \in T S} \xi_{i}+C_{2} \sum_{i \in T S}^{y_{i} \neq 1} \eta_{i} \\
& y_{i}\left(w^{T} x_{i}+b\right) \geq 1-\xi_{i} \quad \forall i \in T S \\
& w^{T} z_{i} \geq 1-\eta_{i} \quad \forall i \in T S: y_{i} \neq 1 \\
& \xi_{i} \geq 0 \quad \forall i \in T S \\
& \eta_{i} \geq 0 \quad \forall i \in T S: y_{i} \neq 1
\end{aligned}
$$

where the vector $z$ is defined as:

$$
z_{i}=x_{\text {trg }_{i}}-x_{i} \quad \forall i \in T S: y_{i} \neq 1
$$

\section{Wolfe Dual of the new training problem}

In order to build the Wolfe Dual of the quadratic optimization problem (24)-(28), it is necessary to introduce the dual multipliers of the constraints:

- $\lambda_{i} \quad \forall i \in T S:$ the multiplier associated to constraints (25)

- $\rho_{i} \quad \forall i \in T S: y_{i} \neq 1$ : the multiplier associated to constraints (26)

- $\mu_{i} \forall i \in T S$ : the multiplier associated to constraints (27)

- $\theta_{i} \quad \forall i \in T S: y_{i} \neq 1$ : the multiplier associated to constraints (28)

Let us define the vector $\lambda$ and $\rho$ as the vectors of size $l_{1}$ and $l_{2}$ respectively containing $\lambda_{i}(\forall i \in T S)$ and $\rho_{i}\left(\forall i \in T S: y_{i} \neq 1\right)$. Then we define the following matrix $\Sigma \in$ $\Re^{(l 1+l 2) \times n}$ : 


$$
\Sigma=\left(\begin{array}{c}
y^{1}\left(x^{1}\right)^{T} \\
\vdots \\
y^{l_{1}}\left(x^{l_{1}}\right)^{T} \\
\left(z^{1}\right)^{T} \\
\vdots \\
\left(z^{l_{2}}\right)^{T}
\end{array}\right)
$$

The following proposition holds:

Proposition 1 The dual problem of problem (24) is

$$
\begin{aligned}
& \min \frac{1}{2}\left(\lambda^{T} \rho^{T}\right) \Sigma \Sigma^{T}\left(\begin{array}{c}
\lambda \\
\rho
\end{array}\right)-e^{T} \lambda-e^{T} \rho \\
& y^{T} \lambda=0 \\
& 0 \leq \lambda \leq C_{1} e \\
& 0 \leq \rho \leq C_{2} e
\end{aligned}
$$

Proof The Wolfe dual of problem (24)-(28) is given by:

$$
\begin{aligned}
\max _{w, b, \lambda, \rho, \mu, \theta} L(w, b, \xi, \eta, \lambda, \rho, \mu, \theta) \\
\nabla_{w} L(w, b, \xi, \eta, \lambda, \rho, \mu, \theta)=0 \\
\nabla_{b} L(w, b, \xi, \eta, \lambda, \rho, \mu, \theta)=0 \\
\nabla_{\xi} L(w, b, \xi, \eta, \lambda, \rho, \mu, \theta)=0 \\
\nabla_{\eta} L(w, b, \xi, \eta, \lambda, \rho, \mu, \theta)=0 \\
\lambda, \rho, \mu, \theta \geq 0
\end{aligned}
$$

where $L(w, b, \xi, \eta, \lambda, \rho, \mu, \theta)$ is the Lagrangian of optimization problem (24)-(28) that can be expressed as follows:

$$
\begin{aligned}
L(w, b, \xi, \eta, \lambda, \rho, \mu, \theta)= & \frac{1}{2}\|w\|^{2}+C_{1} \sum_{i \in T S} \xi_{i}+C_{2} \sum_{i \in T S}^{y_{i} \neq 1} \eta_{i} \\
& -\sum_{i \in T S} \lambda_{i}\left(y_{i}\left(w^{T} x_{i}+b\right)-1+\xi_{i}\right)+ \\
& -\sum_{i \in T S}^{y_{i} \neq 1} \rho_{i}\left(w^{T} z_{i}-1+\eta_{i}\right)-\sum_{i \in T S} \mu_{i} \xi_{i}-\sum_{i \in T S}^{y_{i} \neq 1} \theta_{i} \eta_{i}
\end{aligned}
$$

By rearranging terms equation 39 can be rewritten as:

$$
\begin{aligned}
& L(w, b, \xi, \eta, \lambda, \rho, \mu, \theta)=\frac{1}{2}\|w\|^{2}+\sum_{i \in T S} \xi_{i}\left(C_{1}-\lambda_{i}-\mu_{i}\right)+\sum_{i \in T S} \lambda_{i}+\sum_{i \in T S}^{y_{i} \neq 1} \rho_{i}+ \\
& +\sum_{i \in T S}^{y_{i} \neq 1} \eta_{i}\left(C_{2}-\rho_{i}-\theta_{i}\right)-w^{T}\left(\sum_{i \in T S} \lambda_{i} y_{i} x_{i}+\sum_{i \in T S}^{y_{i} \neq 1} \rho_{i} z_{i}\right)-b \sum_{i \in T S} \lambda_{i} y_{i}
\end{aligned}
$$

The constraints of the Wolfe Dual (equations 34-37) can now be computed based on the Lagrangian function in equation 40 . The equation $\nabla_{w} L(w, b, \xi, \eta, \lambda, \rho, \mu, \theta)=0$ leads to an expression for $w$ : 


$$
w=\left(\sum_{i \in T S} \lambda_{i} y_{i} x_{i}+\sum_{i \in T S}^{y_{i} \neq 1} \rho_{i} z_{i}\right),
$$

whereas the equation $\nabla_{b} L(w, b, \xi, \eta, \lambda, \rho, \mu, \theta)=0$ leads to the constraint

$$
\sum_{i \in T S} \lambda_{i} y_{i}=0
$$

Equation $\frac{\partial L(w, b, \xi, \eta, \lambda, \rho, \mu, \theta)}{\partial \xi_{i}}=0$ allows to derive $\mu_{i}$ as a function of $\lambda$ :

$$
C_{1}-\lambda_{i}-\mu_{i}=0 \quad \forall i \in T S
$$

whereas $\frac{\partial L(w, b, \xi, \eta, \lambda, \rho, \mu, \theta)}{\partial \eta_{i}}=0$ results in an expression of $\theta_{i}$ as a function of $\rho_{i}$

$$
C_{2}-\rho_{i}-\theta_{i}=0 \quad \forall i \in T S: y_{i} \neq 1
$$

Non-negativity of the multipliers $\lambda, \rho, \mu, \theta$ combined with equations (43) and (44) result in the following set of constraints:

$$
\begin{array}{lr}
0 \leq \lambda_{i} \leq C_{1} & \forall i \in T S \\
0 \leq \rho_{i} \leq C_{2} & \forall i \in T S: y_{i} \neq 1
\end{array}
$$

We can plug equations (43) and (44) in the objective function, getting:

$$
\begin{aligned}
& L(w, b, \xi, \eta, \lambda, \rho, \mu, \theta)=\frac{1}{2}\|w\|^{2}+\sum_{i \in T S} \xi_{i}\left(C_{1}-\lambda_{i}-\mu_{i}\right)+\sum_{i \in T S} \lambda_{i}+\sum_{i \in T S}^{y_{i} \neq 1} \rho_{i}+ \\
& +\sum_{i \in T S}^{y_{i} \neq 1} \eta_{i}\left(C_{2}-\rho_{i}-\theta_{i}\right)-w^{T}\left(\sum_{i \in T S} \lambda_{i} y_{i} x_{i}+\sum_{i \in T S}^{y_{i} \neq 1} \rho_{i} z_{i}\right)-b \sum_{i \in T S} \lambda_{i} y_{i}= \\
& =\frac{1}{2}\|w\|^{2}+\sum_{i \in T S} 0 \times \xi_{i}+\sum_{i \in T S} \lambda_{i}+\sum_{i \in T S}^{y_{i} \neq 1} \rho_{i}+\sum_{i \in T S}^{y_{i} \neq 1} 0 \times \eta_{i}-w^{T} w-0 \times b= \\
& =-\frac{1}{2}\|w\|^{2}+\sum_{i \in T S} \lambda_{i}+\sum_{i \in T S}^{y_{i} \neq 1} \rho_{i}
\end{aligned}
$$

The Wolfe Dual of problem (24)-(28) can then be expressed by using equation (47) as objective and equations (41), (42), (45), (46) as constraints.

$$
\begin{aligned}
& \min \frac{1}{2}\|w\|^{2}-\sum_{i \in T S} \lambda_{i}-\sum_{i \in T S}^{y_{i} \neq 1} \rho_{i} \\
& w=\left(\sum_{i \in T S} \lambda_{i} y_{i} x_{i}+\sum_{i \in T S}^{y_{i} \neq 1} \rho_{i} z_{i}\right) \\
& \sum_{i \in T S} \lambda_{i} y_{i}=0 \\
& 0 \leq \lambda_{i} \leq C_{1} \quad \forall i \in T S \\
& 0 \leq \rho_{i} \leq C_{2} \quad \forall i \in T S: y_{i} \neq 1
\end{aligned}
$$


Note that:

$$
\begin{aligned}
& \|w\|^{2}=w^{T} w=\left(\sum_{i \in T S} \lambda_{i} y_{i} x_{i}+\sum_{i \in T S}^{y_{i} \neq 1} \rho_{i} z_{i}\right)^{T}\left(\sum_{i^{\prime} \in T S} \lambda_{i^{\prime}} y_{i^{\prime}} x_{i^{\prime}}+\sum_{i^{\prime} \in T S}^{y_{i^{\prime}} \neq 1} \rho_{i^{\prime}} z_{i^{\prime}}\right)= \\
& \sum_{i \in T S} \sum_{i^{\prime} \in T S}\left(\lambda_{i} \lambda_{i^{\prime}} y_{i} y_{i^{\prime}}\left(x_{i}\right)^{T} x_{i^{\prime}}\right)+\sum_{i \in T S}^{y_{i} \neq 1} \sum_{i^{\prime} \in T S}^{y_{i^{\prime}} \neq 1}\left(\rho_{i} \rho_{i^{\prime}}\left(z_{i}\right)^{T} x_{i^{\prime}}\right)+2 \sum_{i \in T S} \sum_{i^{\prime} \in T S}^{y_{i^{\prime}} \neq 1}\left(\lambda_{i} \rho_{i^{\prime}} y_{i}\left(x_{i}\right)^{T} z_{i^{\prime}}\right)
\end{aligned}
$$

Let us define the vector $\lambda$ and $\rho$ as the vectors of size $l_{1}$ and $l_{2}$ respectively containing $\lambda_{i}(\forall i \in T S)$ and $\rho_{i}\left(\forall i \in T S: y_{i} \neq 1\right)$. Then we define the following matrix $\Sigma \in$ $\Re^{(l 1+l 2) \times n}$ :

$$
\Sigma=\left(\begin{array}{c}
y^{1}\left(x^{1}\right)^{T} \\
\vdots \\
y^{l_{1}}\left(x^{l_{1}}\right)^{T} \\
\left(z^{1}\right)^{T} \\
\vdots \\
\left(z^{l_{2}}\right)^{T}
\end{array}\right)
$$

The dual problem can then be rewritten as

$$
\begin{aligned}
& \min \frac{1}{2}\left(\lambda^{T} \rho^{T}\right) \Sigma \Sigma^{T}\left(\begin{array}{c}
\lambda \\
\rho
\end{array}\right)-e^{T} \lambda-e^{T} \rho \\
& y^{T} \lambda=0 \\
& 0 \leq \lambda \leq C_{1} e \\
& 0 \leq \rho \leq C_{2} e
\end{aligned}
$$

that is still a quadratic convex programming problem.

\section{Algorithmic framework}

In Fig. 2, we summarize the proposed approach, which takes in input the EEG signals of a P300 Speller task for a subject and outputs the performance reached in terms of accuracy and ITR. The same pipeline is used within both the no stopping and the early stopping setting.

This generic framework allows for performing several design choices for both the preprocessing phase and the hyperplane construction. Details on how we preprocessed our datasets are reported in Sect. 6.1. Regarding the hyperplane construction method, we used both the standard SVM problem and the training problem proposed in Sect. 3.

Our framework requires to execute an offline phase in which the hyperplane construction problem is solved and the score vector is computed by using one of the MILP problems proposed in Sects. 2.1 (no stopping setting) and 2.2 (early stopping setting). In the early stopping setting the MILP problem will also output the reliability threshold $\Delta$. Solving the MILP problems requires to partition the decision values of offline data in 5 confidence zones (as specified in Fig. 1) which are retrieved on the basis of the distribution of the decision values. The output of the offline phase is composed both by the computed hyperplane, the scores vector and eventually the threshold $\Delta$. These values are then used in an online scenario in order to evaluate subject's performance both in terms of accuracy (\%) and Information Transfer Rate (bit/min), which is of particularly interest in the early stopping setting. 


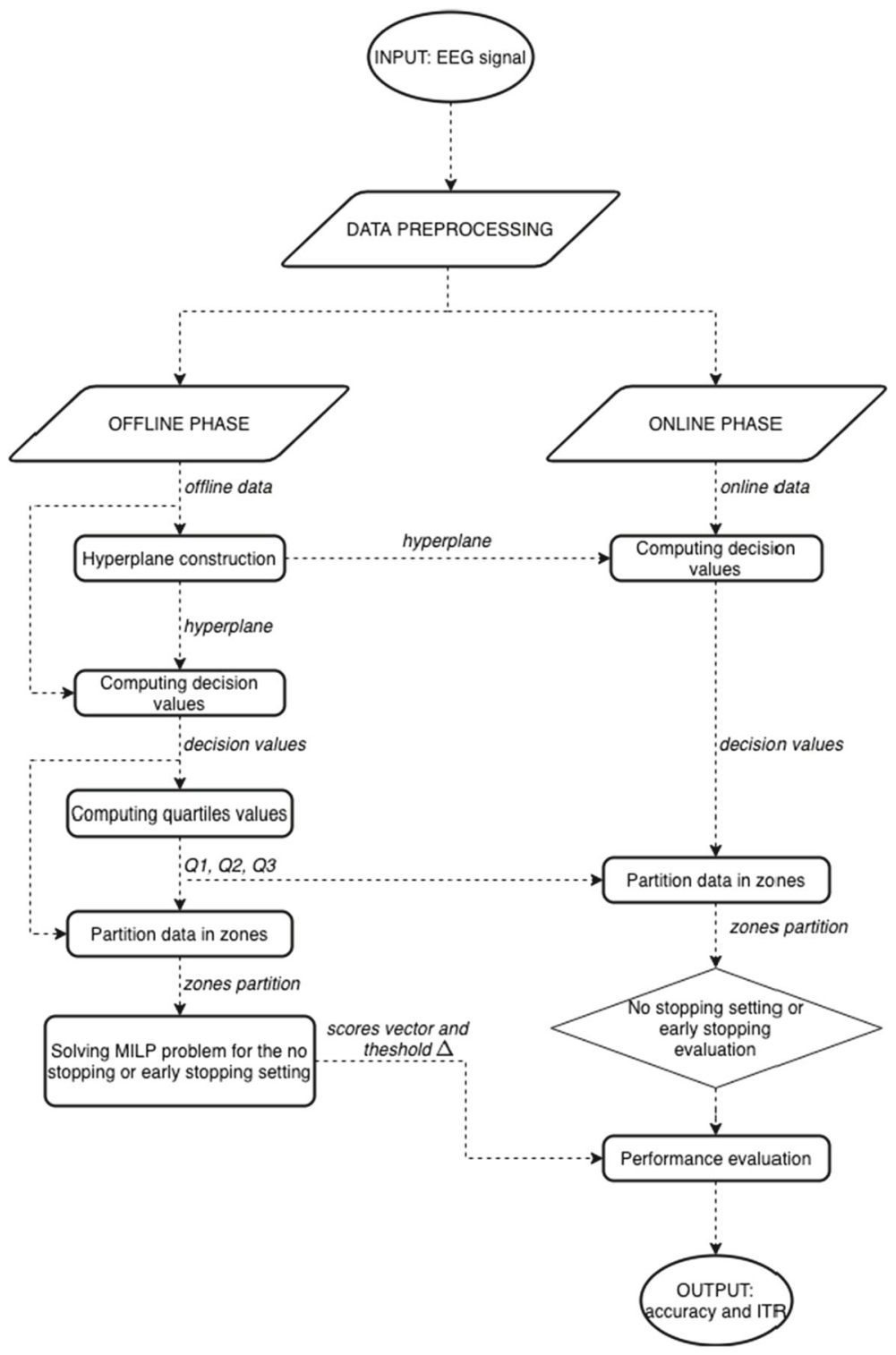

Fig. 2 Flowchart of the proposed algorithmic framework, which takes in input the EEG signal of a subject in a P300 Speller task. The proposed framework requires to perform an offline phase in order to instantiate the process. Results from the offline phase are then used to automatically decode online EEG signals and evaluate the performance results reached by the subject. This pipeline is valid both in a no stopping and early stopping environment; in the latter case, in the online phase the early stopping condition will be evaluated at every incoming iteration before computing the performances obtained for the subject

\section{Numerical results}

\subsection{Dataset}

We tested our approaches on five different datasets: 
(A)

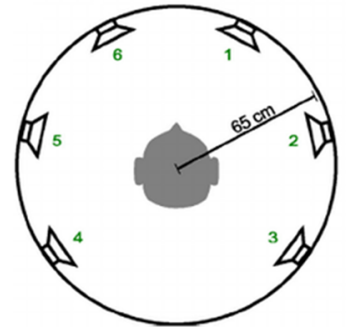

(B)

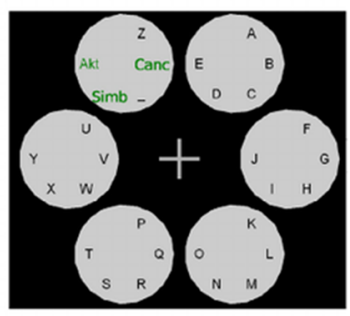

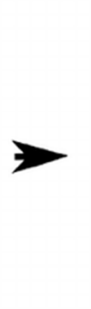

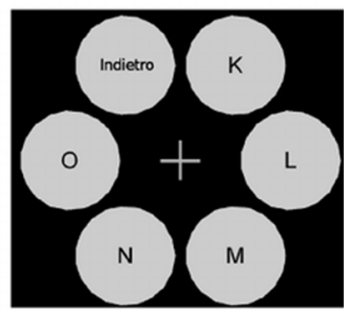

Fig. 3 Graphical representation of the AMUSE paradigm in which six speakers are places all over the subject. In the first level, each speaker is used to represent a set of characters, while on the second level each speaker is used to represent a single character among the previously selected set

AMUSE

P300 Speller

ALS P300 Speller

MVEP

Center Speller

Akimpech
The protocol is based on auditory stimulus elicited by means of spatially located speakers, we have two levels, 15 rounds, six classes for each level, see Fig. 3 (Schreuder et al. 2011). It is performed on healthy subjects and downloadable by the BNCI horizon website http://bncihorizon-2020.eu/database/data-sets.

The protocol is the classical P300 Speller (Farwell and Donchin 1988), performed on 10 healthy subjects.

The protocol is the classical P300 Speller (Farwell and Donchin 1988), performed on 8 patients suffering of Amyotrophic Lateral Sclerosis (ALS).

It is a visual protocol in which a moving pattern generates a movementonset visual evoked potential that is used to recognize the user's choice. This protocol is based on modifications of Cake Speller protocol (Treder et al. 2011). Sixteen healthy subjects have been involved in the study.

It is a visual protocol where we have a visual stimulus elicited by means of three different stimuli, two levels, 10 rounds, six classes for each level (Treder et al. 2011). It is performed on 13 healthy subjects.

It is a P300 Speller performed on 27 healthy subjects, the number of characters is 16 with 15 iterations for each character in the calibration phase, whereas in the online phase changes depending on the subject.

Details of the datasets are reported in Table 2. Please note that we have evaluated our strategy on EEG data recorded from 95 subjects thus assessing its generalization capabilities.

All EEG signals were pre-processed and features were extracted with the NPXLab Suite (Bianchi 2018). Two principal pre-processing operations were applied:

- Electrodes selection: for the datasets Center Speller, MVEP, and AMUSE (see Sect. 6.1) we kept just the electrodes belonging to the 10-20 EEG placement. This strategy allows us to reduce both the dimension of the dataset and the overfitting;

- k-decimation: this technique was applied to all datasets in order to reduce overfitting. In this case, we down-sampled the EEG signal from every electrode by replacing each $k$ consecutive samples with their average value.

Let's recall that the OSBF strategy requires to compute the quartiles of the training set decision values in order to assign scores to stimuli. In this scenario, we stress that, for the standard P300 Speller's paradigm, stimuli corresponding to the intensification of rows and columns are considered separately; in fact, we observed that the distribution of the decision 


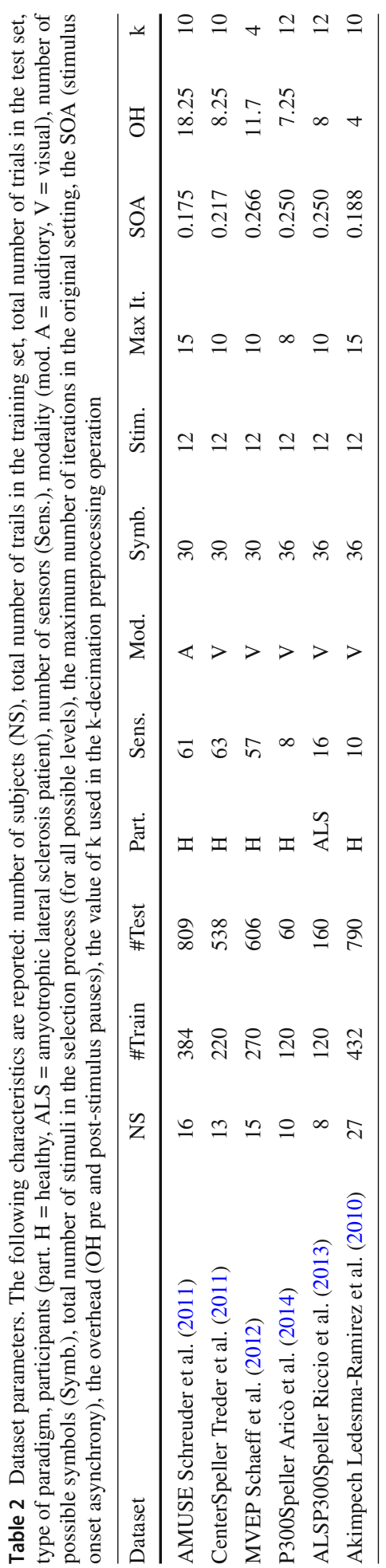


values was different for row and column stimuli. The other paradigms we considered are based on two-levels of selection: in this case, we considered stimuli corresponding to the outer and inner level together for computing the quartiles, since we observed similar distributions of the decision values.

\subsection{No stopping scenario}

As a first step, we evaluate the impact of choosing the scores by solving problem (5). We compare our strategy with both the classical DV avg approach and the SBF decision function (Bianchi et al. 2019) where we sum up the heuristically determined scores for all the available iterations (i.e., we use it in a no stopping fashion). We build the separating hyperplane by training a linear SVM with the package Liblinear (Fan et al. 2008). We try both the L1 and L2 loss, and since there is no clear winner, we report the results obtained with both the losses. Table 3 shows the accuracy-i.e. the percentage of correctly classified characters-obtained by the different approaches. Findings in Table 3 show that the OSBF outperforms the other two approaches since it reaches the highest accuracy on all the datasets. Please note that the OSBF is computationally cheap since the solution of problem (5) is extremely fast (order of few seconds for each MILP problem), and does not require any cross-validation phase (we fixed parameters $C_{1}$ in all the experiments). In order to further improve the accuracy, we try and build the hyperplane by solving the dual problem (33). We call this approach M-SVM. In order to solve problem (33), we apply a modification of the dual coordinate algorithm as described in the "Appendix 1". Also in this case, we do not perform any cross validation but we fix $C_{2}=0.1 \times C_{1}$ and we use the same value of $C_{1}$ of the previous experiment.

A statistical test (Wilcoxon Matched Pair Test, $\mathrm{p}<0.05$ ) performed on the detailed data of Table 13 (see "Appendix 2") indicated that OSBF M-SVM performed better than any other methods after Bonferroni correction, with the only exception of OSBF-L2 SVM, whose difference was statistically significant only before the aforementioned correction for multiple comparisons.

Looking at the average results in Table 4 it emerges that the results obtained by OSBF applied to the M-SVM improve on average only on some datasets, with a significant improvement on the two most difficult datasets: the one containing ALS patients and AMUSE. The intuition was that it could help only when standard SVM is not "good enough". In order to better understand the contribution of the new training problem, we look at the single-subject results, dividing the participants (across all the datasets) into two classes:

Class 1 subjects where the standard SVM problem is better than the new M-SVM; Class 2 subjects where the standard SVM problem is worse than the new M-SVM.

We observed that Class 1 and Class 2 contain respectively about $16 \%$ and $23 \%$ of the subjects among all datasets, whereas in the remaining $61 \%$ of the subjects the standard SVM and the M-SVM perform exactly the same.

In Table 5, we report the average accuracy on both classes, and it is quite evident that the new training problem helps whenever the starting accuracy is not too high. When the starting accuracy is high, the performance does not change or gets worse probably for the overfitting. Interestingly, adding the constraints on the maximum decision value can be interpreted as a form of data augmentation. Indeed, if we include the bias $b$ into the vector $w$, augmenting each data point in the training set with a last component equal to 1 , we can reinterpret the constraints (22) as standard sign constraints imposed on the point $z_{i}$, with label $\hat{y}_{i}=1$. Therefore, we are augmenting our training set by adding the points $z_{i}$, as shown in Fig. 4, and this results in balancing the dataset. 


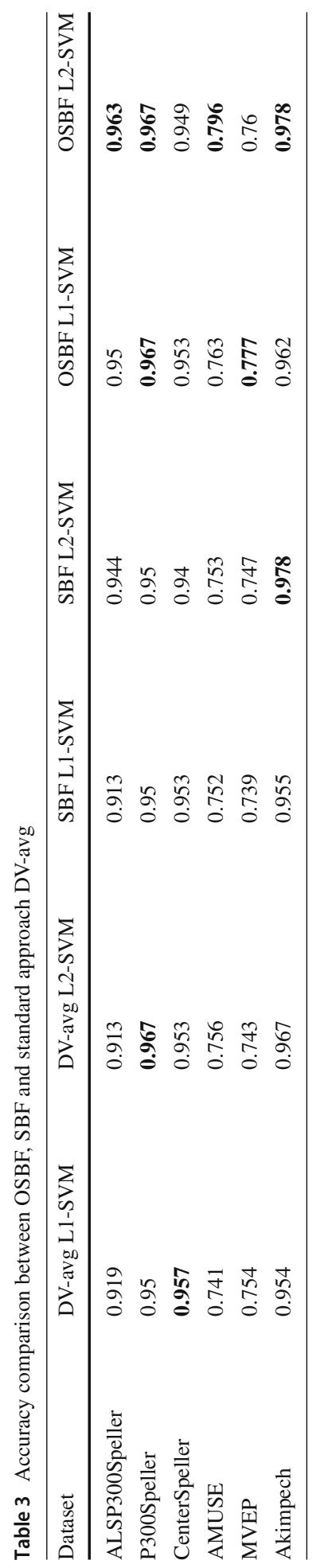


Table 4 Accuracy obtained by OSBF with the different hyperplanes: standard SVM with L1 loss, standard SVM with L2 loss, and the new M-SVM obtained by solving problem (33)

\begin{tabular}{llll}
\hline Dataset & OSBF L1-SVM & OSBF L2-SVM & OSBF M-SVM \\
\hline ALSP300Speller & 0.95 & 0.962 & $\mathbf{0 . 9 7 5}$ \\
P300Speller & $\mathbf{0 . 9 6 7}$ & $\mathbf{0 . 9 6 7}$ & $\mathbf{0 . 9 6 7}$ \\
CenterSpeller & 0.953 & 0.949 & $\mathbf{0 . 9 6}$ \\
AMUSE & 0.763 & 0.796 & $\mathbf{0 . 8 0 6}$ \\
MVEP & 0.777 & 0.76 & $\mathbf{0 . 7 8 3}$ \\
Akimpech & 0.962 & $\mathbf{0 . 9 7 8}$ & 0.974 \\
\hline
\end{tabular}

\subsection{Early stopping scenario}

As a second step, we consider the early stopping version of both the SBF (that is the current state of the art for early stopping methods) and the OSBF. In order to evaluate the performance of the proposed method with respect to the number of iterations needed for an accurate classification also the theoretical Information transfer rate (ITR, bit/min) has been computed. The ITR is a communication measure based on Shannon channel theory with some simplifying assumptions. It can be computed by dividing the number of bits transmitted per trial (or bit rate, bits/trial) by the trial duration in minute. We compute the bit-rate, using the definition proposed in Wolpaw et al. (1998), as:

$$
\mathrm{B}=\log _{2} N+P \log _{2} P+(1-P) \log _{2} \frac{(1-P)}{(N-1)},
$$

where $N$ is the number of possible symbols in the speller grid and $P$ is the probability that the target symbol is accurately classified at the end of a trial. From (57) the ITR is computed as:

$$
\mathrm{ITR}=\frac{B}{\text { trial duration }}
$$

where

$$
\text { trial duration }=\frac{\mathrm{SOA} \cdot f_{s} \cdot \bar{i}}{60} \min
$$

In (59), SOA refers to the stimulus-onset asynchrony; $f_{s}$ represents the number of stimuli in each stimulation sequence and $\bar{i}$ is the mean number of used iterations to select a symbol. In Tables $6,7,8$ and 9 the results obtained with the early stopping setting are shown. Findings in Table 6 further corroborates the potentials of the OSBF since its outperforms the SBF, no matter what hyperplane is used. In Table 7 we compare the early stopping results in terms of accuracy obtained with the OSBF with the different hyperplanes (L1-SVM, L2-SVM and M-SVM): we can notice that, in this case, the M-SVM reaches a higher level of accuracy than the other methods among almost all datasets. Tables 8 and 9 show the results in terms of theoretical ITR. In this case, we can see that all the strategies reach comparable results and there is not a clear winner. This is confirmed by the statistical analysis (Wilcoxon test) which did not reveal any significant difference among the various methods when applied to the data contained on either Tables 14 and 15 included in "Appendix 2". We can then conclude that the OSBF strategy is a more conservative approach than the SBF, since it manages to keep a high level of accuracy preserving the communication speed. 


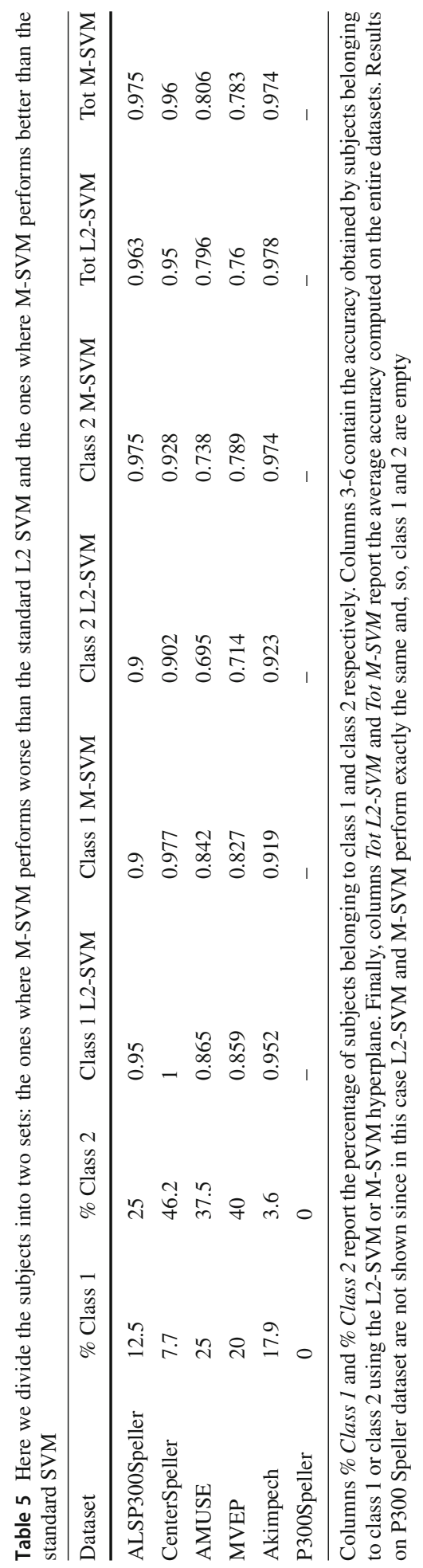




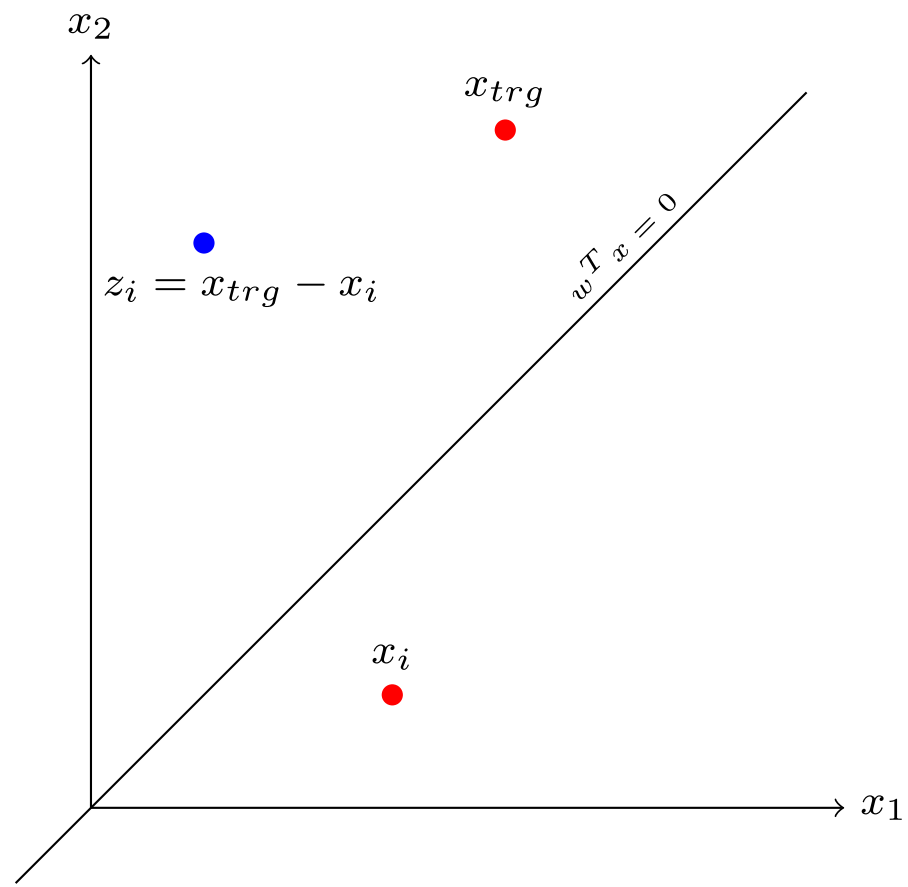

Fig. 4 Given the point $x_{i}$ with label $y_{i}=-1$, it is possible to reinterpret constraints (22) as adding to the training set the points $z_{i}$ with label $\hat{y}_{i}=1$. Please note that this is a simplified example where samples are represented with 2-dimensional data points with features $x_{1}$ and $x_{2}$

Table 6 Accuracy obtained in the early stopping setting by the SBF and OSBF using the separation hyperplane given by a linear SVM with both L1 and L2 losses

\begin{tabular}{lllll}
\hline Dataset & SBF L1-SVM & SBF L2-SVM & OSBF L1-SVM & OSBF L2-SVM \\
\hline ALSP300Speller & 0.85 & 0.863 & $\mathbf{0 . 9 2 5}$ & $\mathbf{0 . 9 2 5}$ \\
P300Speller & 0.95 & 0.95 & $\mathbf{0 . 9 6 7}$ & 0.95 \\
CenterSpeller & 0.903 & 0.893 & $\mathbf{0 . 9 4 4}$ & 0.931 \\
AMUSE & 0.636 & 0.647 & $\mathbf{0 . 7 5 6}$ & 0.744 \\
MVEP & 0.712 & 0.712 & $\mathbf{0 . 7 4 8}$ & 0.744 \\
Akimpech & 0.911 & 0.934 & 0.943 & $\mathbf{0 . 9 4 8}$ \\
\hline
\end{tabular}

As a final analysis, in Tables 10, 11 and 12 we compare the no stopping and early stopping configurations respectively with L1-SVM, L2-SVM and M-SVM hyperplanes. It is reasonable to expect that the early stopping setting leads to a increase of ITR; on the other hand, we can expect a loss in terms of accuracy. In order to evaluate these phenomena, we report the ITR levels obtained both in the no stopping and early stopping setting and we compute both the percentage of increase in terms of ITR and the percentage of loss in terms of accuracy. We can notice that, no matter what hyperplane is used, the early stopping configuration always leads to a consistent increase in terms of ITR with a small percentage of accuracy loss. 
Table 7 Accuracy obtained in the early stopping setting by the OSBF with the different hyperplanes: standard SVM with L1 loss, standard SVM with L2 loss, and the new M-SVM obtained by solving problem (33)

\begin{tabular}{llll}
\hline Dataset & OSBF L1-SVM & OSBF L2-SVM & OSBF M-SVM \\
\hline ALSP300Speller & 0.925 & 0.925 & $\mathbf{0 . 9 4 4}$ \\
P300Speller & $\mathbf{0 . 9 6 7}$ & 0.95 & $\mathbf{0 . 9 6 7}$ \\
CenterSpeller & 0.944 & 0.931 & $\mathbf{0 . 9 5}$ \\
AMUSE & 0.756 & 0.744 & $\mathbf{0 . 7 6}$ \\
MVEP & 0.748 & 0.744 & $\mathbf{0 . 7 6 0}$ \\
Akimpech & 0.943 & $\mathbf{0 . 9 4 8}$ & 0.942 \\
\hline
\end{tabular}

Table 8 ITR (bit/min) obtained in the early stopping setting by the SBF and OSBF using the separation hyperplan given by a linear SVM with both L1 and L2 losses

\begin{tabular}{lllll}
\hline Dataset & SBF L1-SVM & SBF L2-SVM & OSBF L1-SVM & OSBF L2-SVM \\
\hline ALSP300Speller & 20.187 & 19.234 & 20.187 & $\mathbf{2 0 . 7 1 6}$ \\
P300Speller & $\mathbf{3 4 . 3 8}$ & 34.086 & 32.343 & 30.356 \\
CenterSpeller & $\mathbf{2 8 . 3 7 2}$ & 28.042 & 27.76 & 27.649 \\
AMUSE & 12.67 & 12.929 & 14.490 & $\mathbf{1 5 . 2 3 2}$ \\
MVEP & 9.434 & 9.252 & 10.245 & $\mathbf{1 0 . 6 2 6}$ \\
Akimpech & 34.593 & $\mathbf{3 8 . 4 6 4}$ & 35.061 & 36.532 \\
\hline
\end{tabular}

Table 9 ITR ( bit/min) obtained in the early stopping setting by the OSBF with the different hyperplanes: standard SVM with L1 loss, standard SVM with L2 loss, and the new M-SVM obtained by solving problem (33)

\begin{tabular}{llll}
\hline Dataset & OSBF L1-SVM & OSBF L2-SVM & OSBF M-SVM \\
\hline ALSP300Speller & 20.187 & 20.716 & $\mathbf{2 1 . 7 9}$ \\
P300Speller & $\mathbf{3 2 . 3 4 3}$ & 30.356 & 32.11 \\
CenterSpeller & 27.76 & 27.649 & $\mathbf{2 7 . 9 0 9}$ \\
AMUSE & 14.490 & 15.232 & $\mathbf{1 5 . 3 5}$ \\
MVEP & 10.245 & $\mathbf{1 0 . 6 2 6}$ & 10.431 \\
Akimpech & 35.061 & $\mathbf{3 6 . 5 3 2}$ & 34.811 \\
\hline
\end{tabular}

Table 10 Comparison of the ITR ( bit $/ \mathrm{min}$ ) between the no stopping and early stopping setting for the OSBF decision function by using the standard SVM training problem with L1 loss

\begin{tabular}{lllll}
\hline Dataset & OSBF L1-SVM NS & OSBF L1-SVM ES & ITR increase (\%) & Accuracy loss (\%) \\
\hline ALSP300Speller & 9.342 & 20.187 & 116.1 & 2.6 \\
P300Speller & 12.172 & 32.343 & 165.7 & 0.0 \\
CenterSpeller & 10.281 & 27.760 & 170.0 & 0.9 \\
AMUSE & 5.895 & 14.490 & 145.8 & 0.9 \\
MVEP & 5.940 & 10.245 & 72.5 & 3.8 \\
Akimpech & 10.077 & 35.061 & 247.9 & 2.0 \\
\hline
\end{tabular}


Table 11 Comparison of the ITR ( bit $/ \mathrm{min}$ ) between the no stopping and early stopping setting for the OSBF decision function by using the standard SVM training problem with L2 loss

\begin{tabular}{lllll}
\hline Dataset & OSBF L2-SVM NS & OSBF L2-SVM ES & ITR increase (\%) & Accuracy loss (\%) \\
\hline ALSP300Speller & 9.452 & 20.716 & 119.2 & 3.9 \\
P300Speller & 12.172 & 30.356 & 149.4 & 1.7 \\
CenterSpeller & 10.240 & 27.649 & 170.0 & 2.0 \\
AMUSE & 6.276 & 15.232 & 142.7 & 6.5 \\
MVEP & 5.723 & 10.626 & 85.7 & 2.0 \\
Akimpech & 10.370 & 36.532 & 252.3 & 3.1 \\
\hline
\end{tabular}

Table 12 Comparison of the ITR ( $\mathrm{bit} / \mathrm{min}$ ) between the no stopping and early stopping setting for the OSBF decision function by using the M-SVM training problem

\begin{tabular}{lllll}
\hline Dataset & OSBF M-SVM NS & OSBF M-SVM ES & ITR increase (\%) & Accuracy loss (\%) \\
\hline ALSP300Speller & 9.823 & 21.790 & 121.8 & 3.2 \\
P300Speller & 12.172 & 32.110 & 163.8 & 0.0 \\
CenterSpeller & 10.439 & 27.909 & 167.4 & 1.0 \\
AMUSE & 6.367 & 15.350 & 141.1 & 5.8 \\
MVEP & 6.002 & 10.431 & 73.8 & 2.9 \\
Akimpech & 10.278 & 34.811 & 238.7 & 3.3 \\
\hline
\end{tabular}

\section{Conclusions}

BCIs are proposed for a wide range of applications, such as those for communicating (Sellers et al. 2006), for entertainment (Bianchi 2020), environmental or neuroprostheses control (Muller-Putz and Pfurtscheller 2008), rehabilitation (Bockbrader et al. 2018), and supporting diagnoses (Lugo et al. 2016), to name a few. Moreover, the same paradigm, such as the P300 discussed in this manuscript, can be used in all of them. Despite this large scenario, all of these applications try to get one among these two goals:

(i) to improve the accuracy of the systems, which usually is equivalent to minimize the occurrence of classification errors;

(ii) to maximize the communication speed or, in a more general sense, the information transfer rate.

These two strategies are usually pursued because the consequences of errors are different such as in the cases of a BCI used to drive a wheelchair, as compared to a BCI used to communicate: in the first case an error can harm a user, while in the second case it only leads to a typo. In any case, depending on the application, the strategies to achieve such goals can vary a lot.

This paper focuses on the classification problem that arises in many BCI protocols. The idea was to exploit the knowledge on the protocol in order to improve the classification accuracy and the communication speed of the BCI, that is to achieve both goals or at least to shorten the distance among them, thus allowing a painless tuning of a BCI according to the target application.

The novelty of our approach is three-fold. First, for each participant we determine the optimal scores by solving an optimization problem on her/his training data; secondly, we 
implement an efficient early stopping method by solving a modified version of the optimization problem connected to training; finally, we successfully include the information on the decision function (i.e. the target always is the stimulus having maximum decision value) into the training problem. The main advantage of our proposed methodology is that the preliminary calibration phase becomes completely automatic so that a cross validation phase or manual parameters tuning is no longer fundamental.

Our method achieves such results by means of two main ingredients:

(i) the use of a MILP problem to assign a 'reliability score' to the classification of each stimulus in every iteration

(ii) the definition of a new training problem that keeps into account that the target class is assigned to the stimulus having the maximum decision value.

Both these elements have been applied in two different scenarios: a first one where accuracy was the main focus and all the iterations available for each subject were used both in the calibration and the online phase; a second one where the focus was to improve the communication speed, and hence an early stopping strategy was implemented in the online phase. In order to evaluate the approaches we conducted an extensive experimentation on datasets coming from different protocols and including both healthy subjects and ALS patients. The results show how we were able to improve accuracy and ITR on all the datasets, proving once more that combining machine learning tools to problem knowledge can significantly improve performances.

To our knowledge, according to the literature, it is the first time that a methods was successfully used and performed better than any other in either accuracy and information transfer rate. Moreover, this was verified on six different publicly available datasets, which include either healthy subjects or ALS patients. This remarkable result, which has been obtained through offline analysis, once verified also during online recording sessions, may represents the new gold standard in P300 based BCI paradigms and provide a significant improvement for all the applications that make use of it.

The use of an optimization problem to define how reliable a classification is, can be used also in the context of collaborative BCIs (Poli et al. 2014), where a group decision is made on the basis of the EEG signals of a certain number of subjects. The idea is to use an optimization problem to assign a score to each subject for each trial of a certain task as for example recognizing a face in an image containing a crowded environment (Valeriani and Poli 2019). In this case, the idea is to evaluate from the EEG the reliability of the subject on that task using an automated process based again on a the solution of a MILP problem. This is material of future work.

Funding Open Access funding provided by Universitá degli Studi di Roma Tor Vergata

Open Access This article is licensed under a Creative Commons Attribution 4.0 International License, which permits use, sharing, adaptation, distribution and reproduction in any medium or format, as long as you give appropriate credit to the original author(s) and the source, provide a link to the Creative Commons licence, and indicate if changes were made. The images or other third party material in this article are included in the article's Creative Commons licence, unless indicated otherwise in a credit line to the material. If material is not included in the article's Creative Commons licence and your intended use is not permitted by statutory regulation or exceeds the permitted use, you will need to obtain permission directly from the copyright holder. To view a copy of this licence, visit http://creativecommons.org/licenses/by/4.0/. 


\section{Appendix}

\section{Dual Coordinate Descent Algorithm}

In this section we describe how we modified the Dual Coordinate Descent Algorithm proposed in Hsieh et al. (2008) in order to find the separating hyperplane for problem 24-28. The Dual Coordinate Descent Algorithm basically solves the dual problem applying a Gauss Seidel decomposition method where each variable constitutes a block, and the subproblem with respect to a single variable is globally solved analytically. We adapt the algorithm by modifying the following points:

- how the gradient of the objective function is computed;

- how the hyperplane is updated.

In particular, we can write the objective function $f$ and the separating hyperplane $w$ as:

$$
\begin{aligned}
f= & \frac{1}{2} \sum_{i=1}^{l_{1}} \sum_{j=1}^{l_{1}} \lambda_{i} \lambda_{j} y_{i} y_{j} x_{i}^{T} x_{j}+\sum_{i=1}^{l_{1}} \sum_{j=1}^{l_{2}} \lambda_{i} \rho_{j} y_{i} x_{i}^{T} z_{j} \\
& +\frac{1}{2} \sum_{i=1}^{l_{2}} \sum_{j=1}^{l_{2}} \rho_{i} \rho_{j} z_{i} z_{j}-\sum_{i=1}^{l_{1}} \lambda_{i}-\sum_{i=1}^{l_{2}} \rho_{j} \\
w= & \sum_{i=1}^{l_{1}} \lambda_{i} y_{i} x_{i}+\sum_{i=1}^{l_{2}} \rho_{i} z_{i}
\end{aligned}
$$

Let's define the vector $\alpha=\left[\lambda^{T} \rho^{T}\right] \in \mathbb{R}^{l_{1} \times l_{2}}$. Equations 60 and 61 can equivalently be defined with respect to vector $\alpha$. We can then express the $i$-th component of the gradient of $f(\alpha)$ as:

$$
\nabla_{i} f=\left\{\begin{array}{l}
\sum_{j=1}^{l_{1}} \alpha_{j} y_{i} y_{j} x_{i}^{T} x_{j}+\sum_{j=l_{1}}^{l_{1}+l_{2}} \alpha_{j} y_{i} x_{i}^{T} z_{j}-1 \quad \text { if } i<l_{1} \\
\sum_{j=1}^{l_{1}} \alpha_{j} y_{j} x_{j}^{T} z_{j}+\sum_{j=l_{1} 1}^{l_{1}+l_{2}} \alpha_{j} z_{i}^{T} z_{j}-1 \quad \text { otherwise }
\end{array}\right.
$$

which can be rewritten as:

$$
\nabla_{i} f=\left\{\begin{array}{lr}
y_{i} w^{T} x_{i}-1 & \text { if } i<l_{1} \\
w^{T} z_{i}-1 & \text { otherwise }
\end{array}\right.
$$

We note that in principle we may use liblinear if we include the bias $b$ into the vector $w$, augmenting each data point in the training set with a last component equal to 1 , and we set $C_{1}=C_{2}$. However, it turns out from the experiments that is more effective to treat the $z_{i}$ separately defining a dedicated parameter $C_{2}$ to be chosen in cross validation, which is why we defined our own training algorithm. 


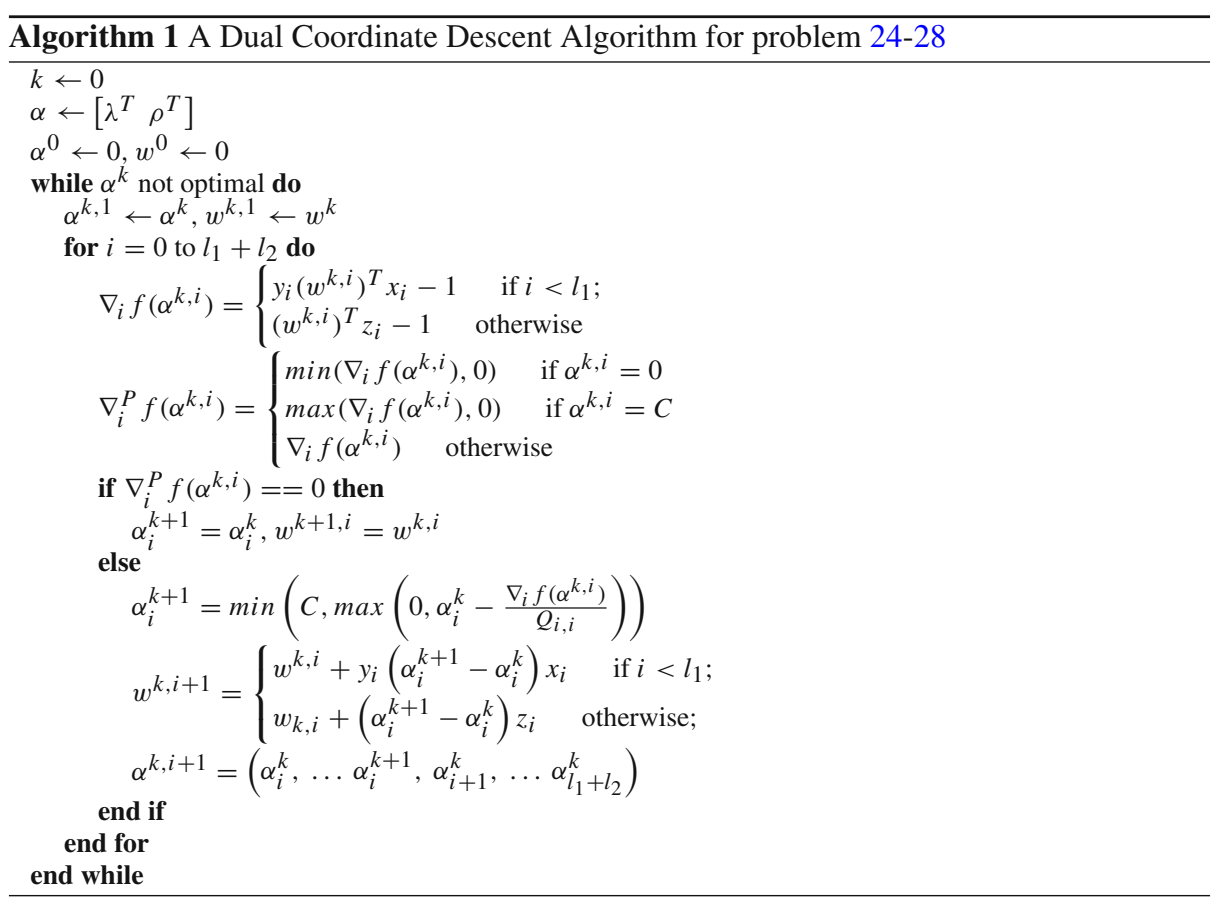

\section{Detailed numerical results}

As a supplement, we provide the detailed results obtained for all subjects for all considered datasets. In Table 13 we provide the results obtained in the no stopping setting, while in Tables 14 and 15 the results for the early stopping setting are reported. 


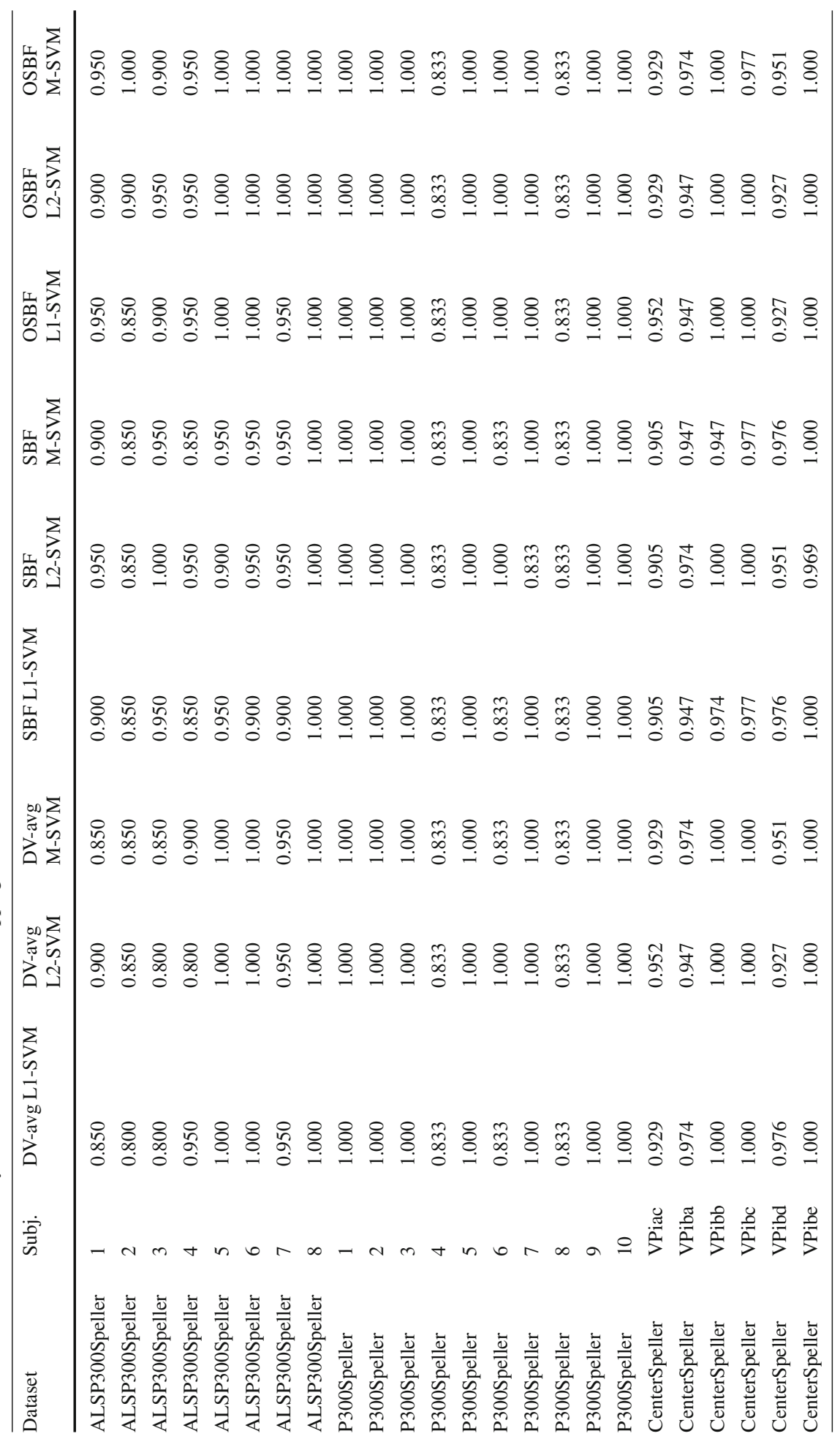




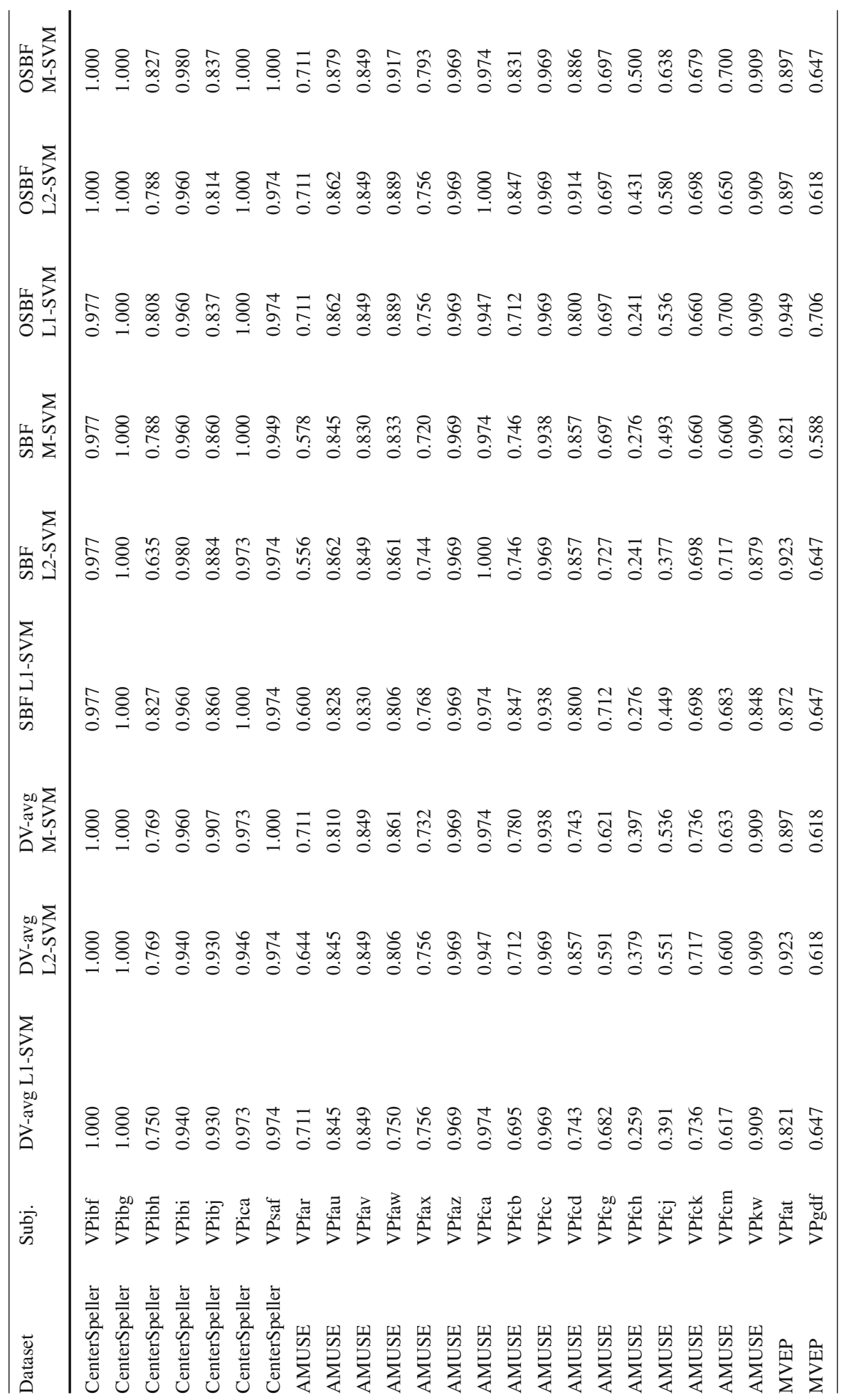




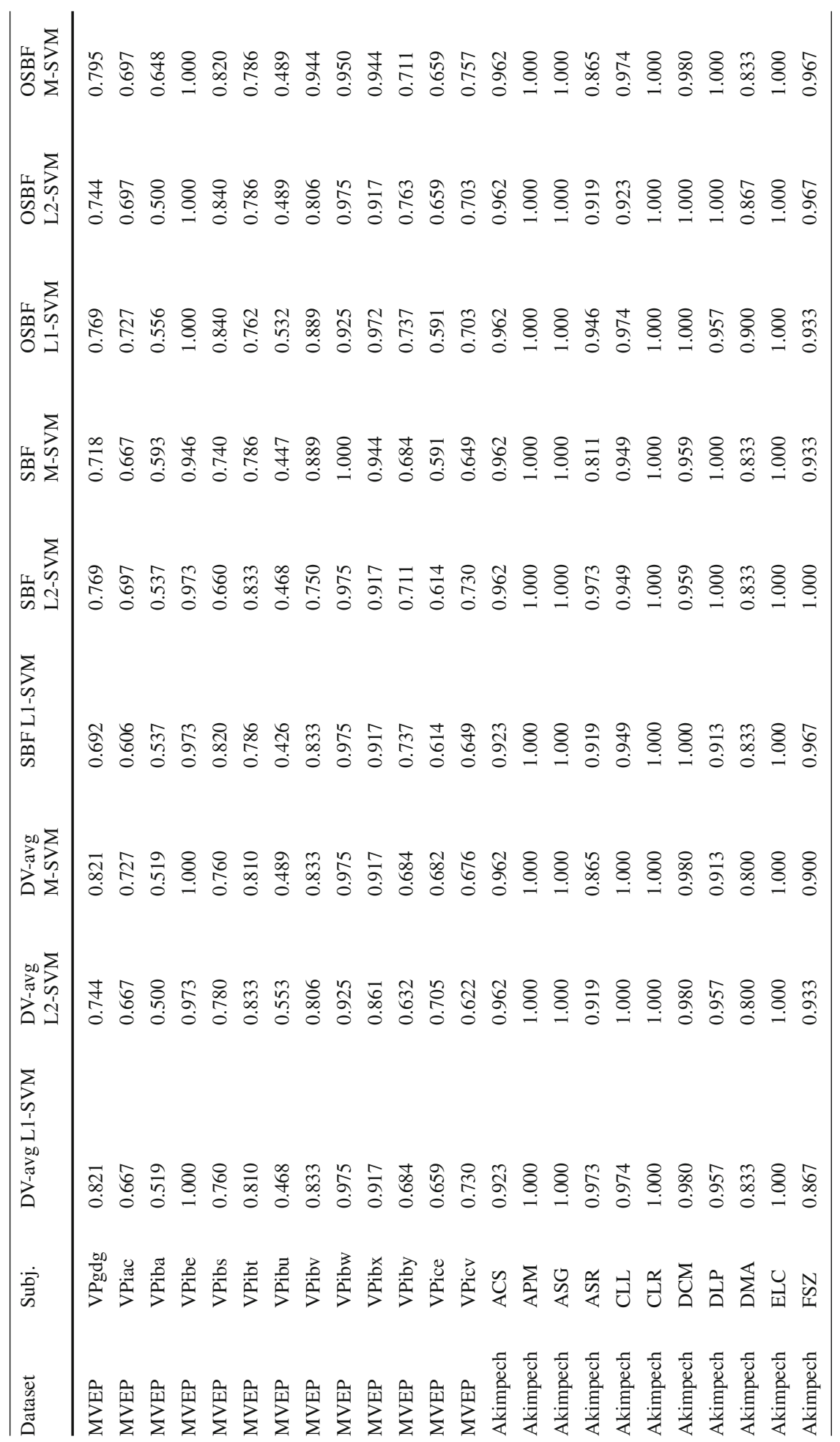




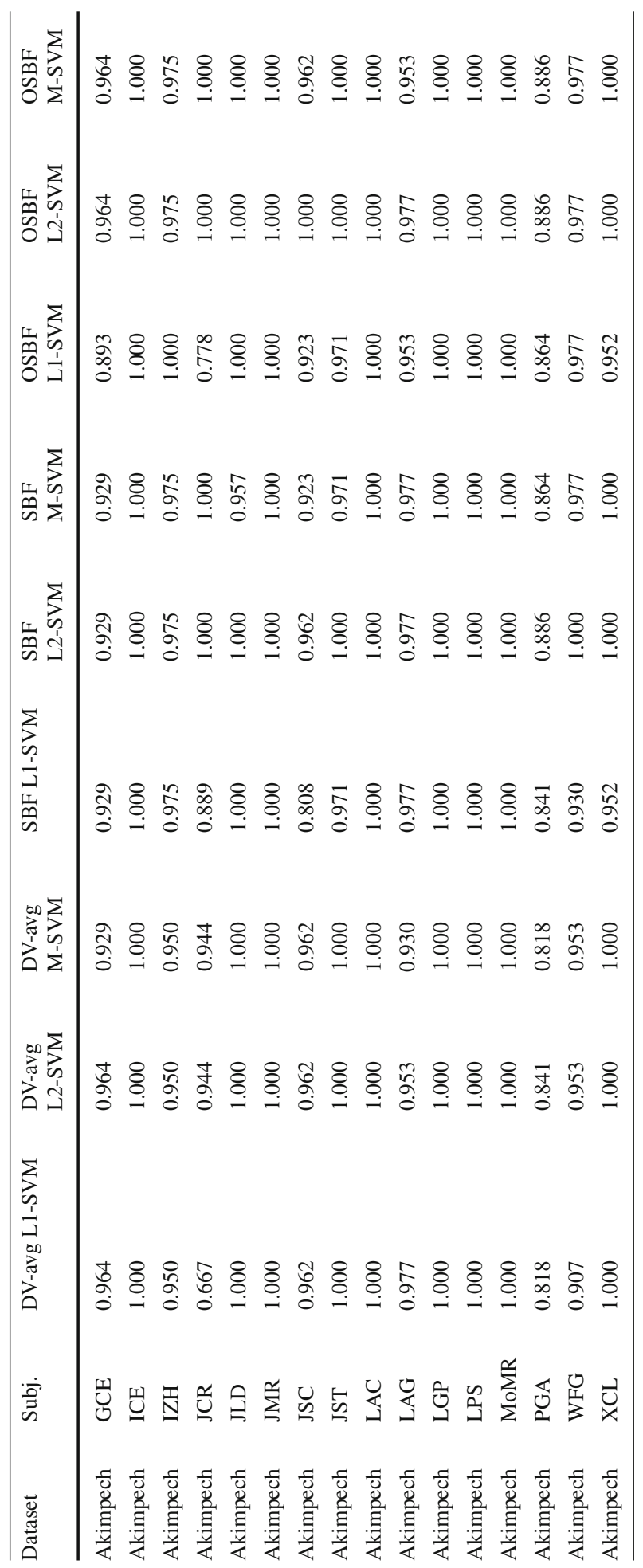

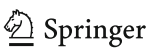




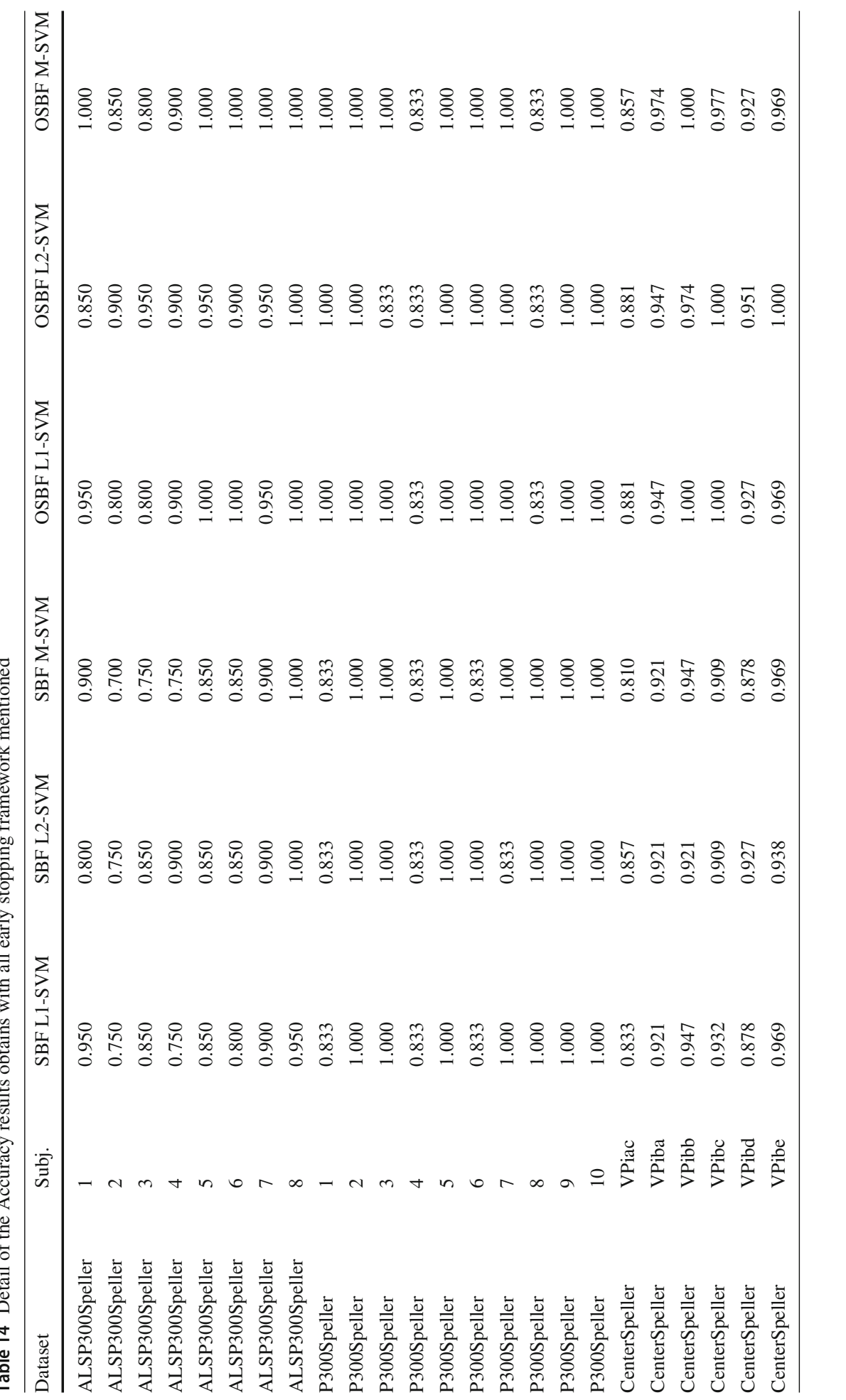




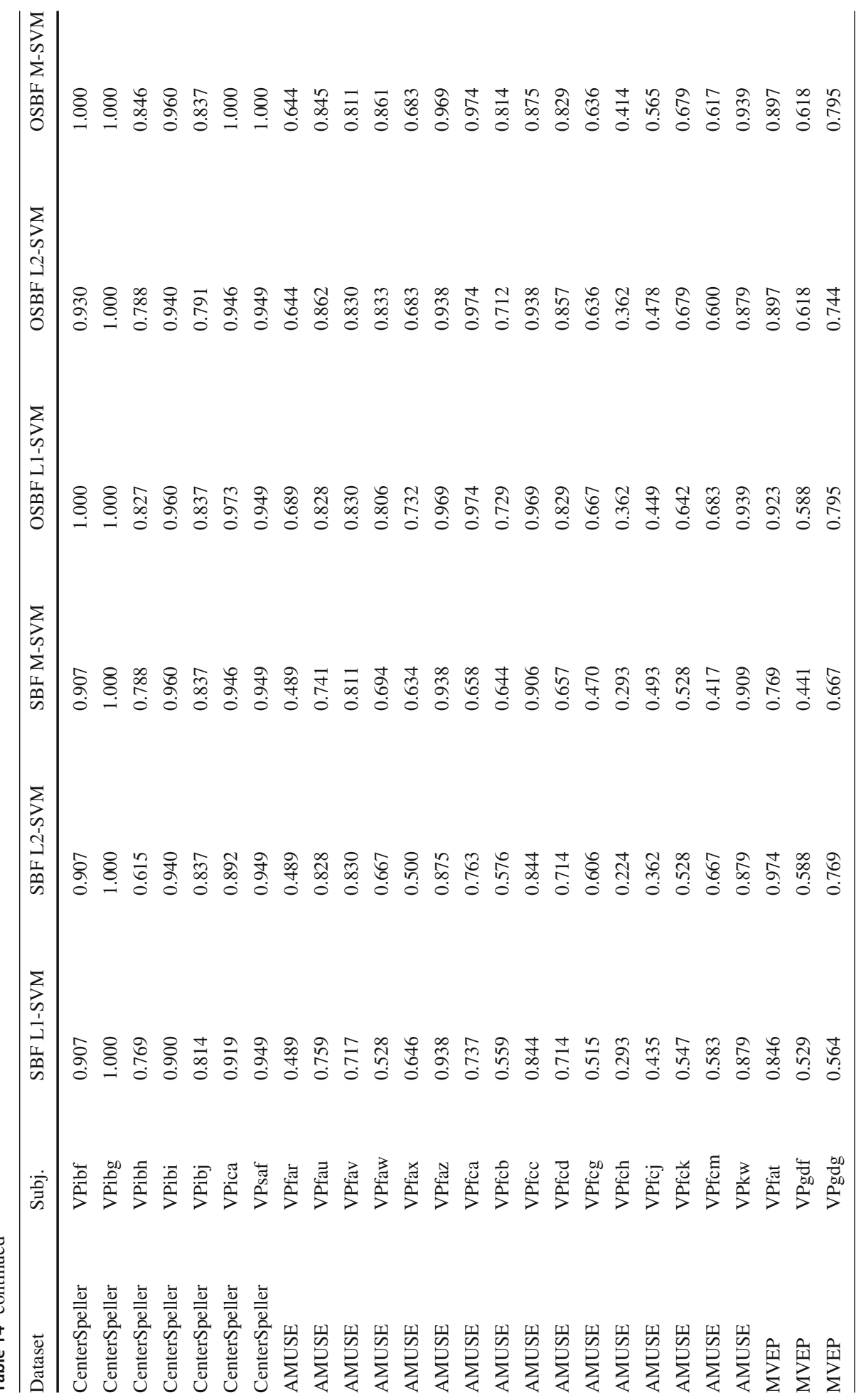




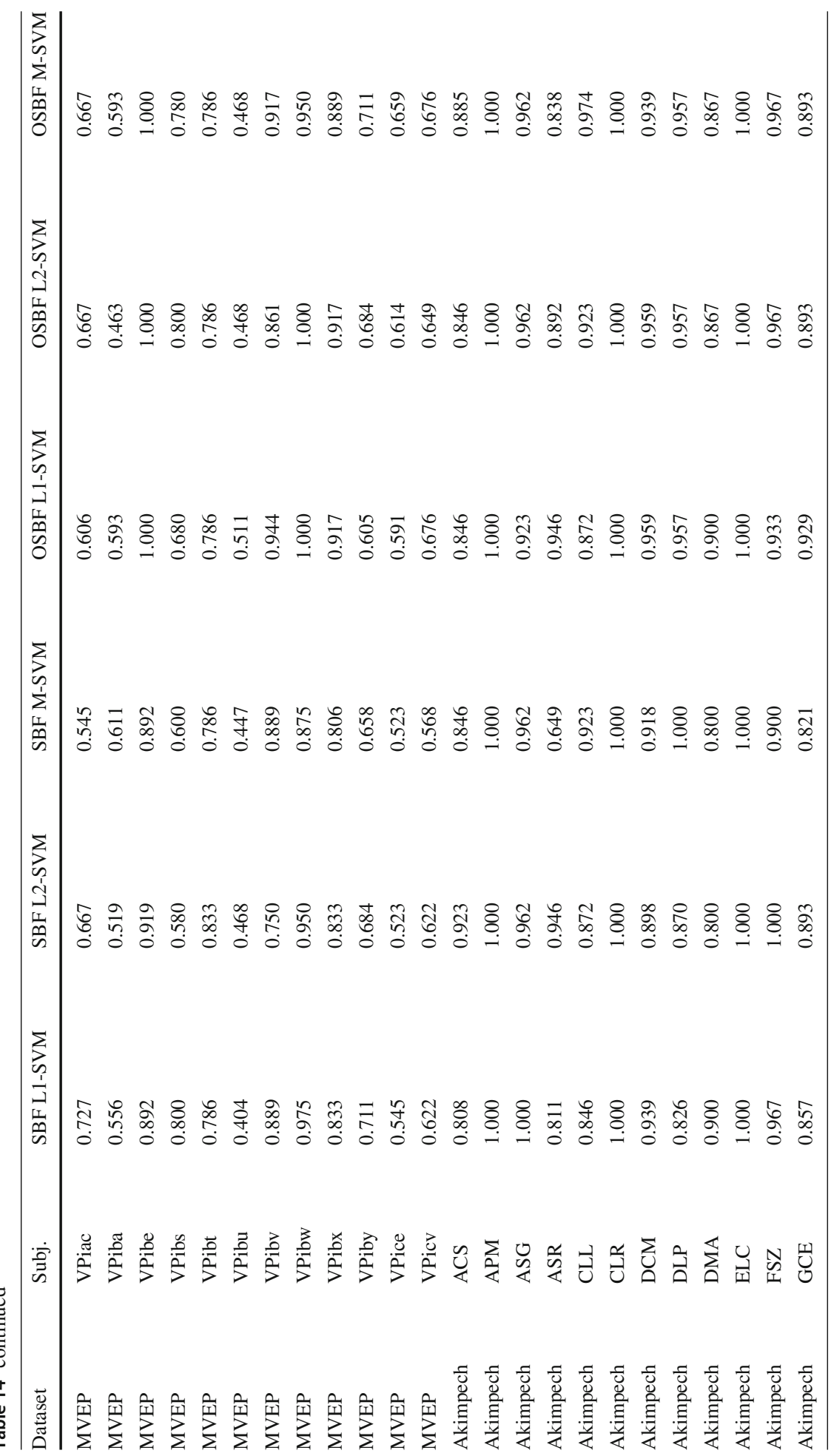




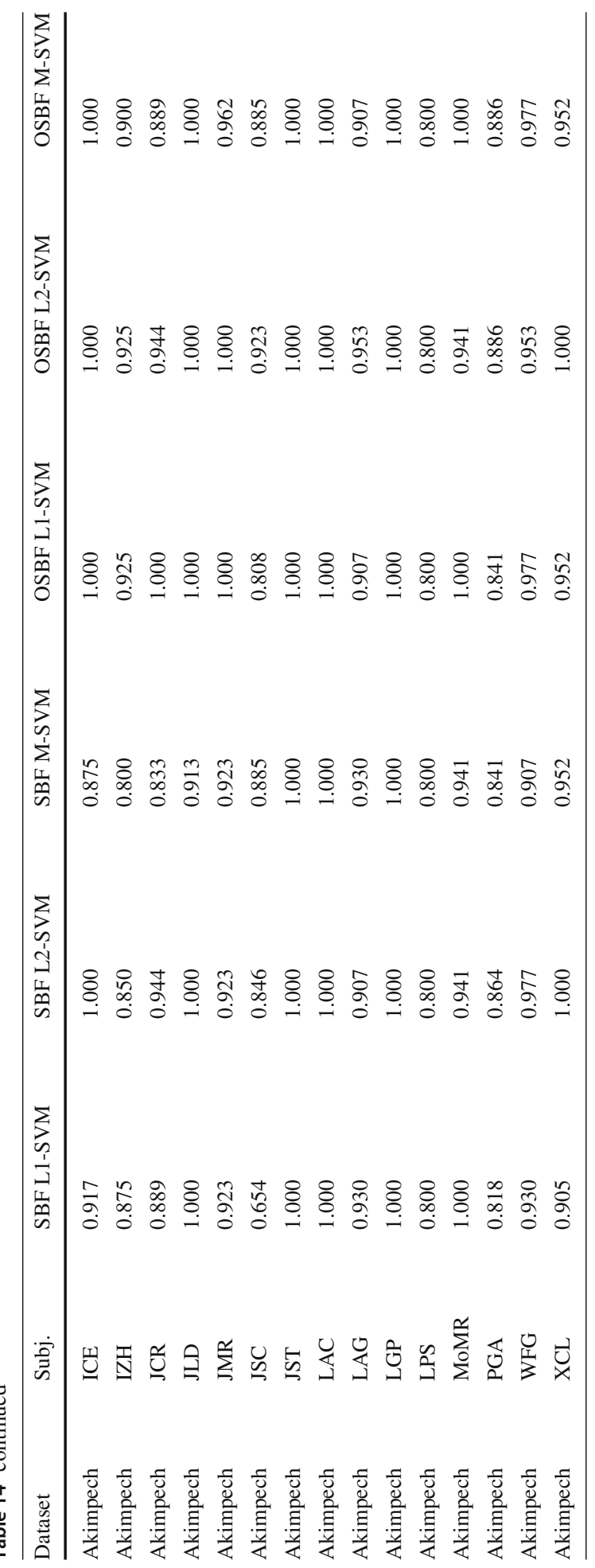

暨 Springer 
Table 15 Detail of the ITR ( it $/ \mathrm{min}$ ) results obtains with all early stopping framework mentioned

\begin{tabular}{|c|c|c|c|c|c|c|c|}
\hline Dataset & Subj. & $\begin{array}{l}\text { SBF } \\
\text { L1-SVM }\end{array}$ & $\begin{array}{l}\text { SBF } \\
\text { L2-SVM }\end{array}$ & $\begin{array}{l}\text { SBF } \\
\text { M-SVM }\end{array}$ & $\begin{array}{l}\text { OSBF } \\
\text { L1-SVM }\end{array}$ & $\begin{array}{l}\text { OSBF } \\
\text { L2-SVM }\end{array}$ & $\begin{array}{l}\text { OSBF } \\
\text { M-SVM }\end{array}$ \\
\hline ALSP300Speller & 1 & 16.599 & 15.734 & 19.593 & 16.599 & 15.239 & 20.175 \\
\hline ALSP300Speller & 2 & 12.222 & 12.556 & 11.111 & 12.222 & 13.845 & 13.722 \\
\hline ALSP300Speller & 3 & 16.492 & 19.073 & 16.298 & 16.492 & 21.032 & 15.210 \\
\hline ALSP300Speller & 4 & 16.424 & 12.501 & 13.826 & 16.424 & 15.879 & 16.836 \\
\hline ALSP300Speller & 5 & 20.577 & 20.771 & 21.207 & 20.577 & 20.565 & 22.236 \\
\hline ALSP300Speller & 6 & 21.654 & 19.193 & 18.605 & 21.654 & 20.305 & 22.356 \\
\hline ALSP300Speller & 7 & 18.508 & 21.477 & 23.429 & 18.508 & 20.565 & 23.236 \\
\hline ALSP300Speller & 8 & 39.018 & 32.566 & 36.928 & 39.018 & 38.296 & 40.548 \\
\hline P300Speller & 1 & 25.132 & 23.148 & 25.132 & 31.020 & 30.263 & 31.815 \\
\hline P300Speller & 2 & 38.774 & 40.025 & 40.025 & 41.359 & 40.025 & 41.359 \\
\hline P300Speller & 3 & 32.652 & 22.977 & 30.263 & 31.815 & 19.547 & 29.542 \\
\hline P300Speller & 4 & 33.831 & 36.650 & 36.650 & 24.433 & 25.132 & 24.433 \\
\hline P300Speller & 5 & 41.359 & 44.314 & 41.359 & 37.599 & 37.599 & 37.599 \\
\hline P300Speller & 6 & 25.132 & 31.020 & 26.655 & 27.573 & 29.542 & 29.542 \\
\hline P300Speller & 7 & 27.573 & 21.454 & 27.573 & 28.855 & 26.974 & 27.573 \\
\hline P300Speller & 8 & 30.263 & 28.855 & 31.020 & 21.990 & 19.991 & 20.456 \\
\hline P300Speller & 9 & 41.359 & 42.786 & 41.359 & 34.466 & 34.466 & 34.466 \\
\hline P300Speller & 10 & 47.722 & 49.631 & 47.722 & 44.314 & 40.025 & 44.314 \\
\hline CenterSpeller & VPiac & 20.249 & 26.589 & 23.718 & 23.546 & 24.404 & 21.732 \\
\hline CenterSpeller & VPiba & 26.307 & 26.794 & 26.794 & 27.868 & 26.982 & 30.014 \\
\hline CenterSpeller & VPibb & 31.684 & 31.049 & 31.816 & 33.617 & 30.699 & 32.973 \\
\hline CenterSpeller & VPibc & 29.426 & 27.713 & 28.289 & 30.473 & 31.336 & 29.083 \\
\hline CenterSpeller & VPibd & 23.146 & 27.908 & 24.662 & 23.647 & 26.704 & 23.576 \\
\hline CenterSpeller & VPibe & 36.558 & 33.161 & 37.370 & 32.653 & 33.707 & 32.973 \\
\hline CenterSpeller & VPibf & 32.471 & 26.430 & 31.821 & 32.140 & 30.004 & 31.824 \\
\hline CenterSpeller & VPibg & 43.509 & 43.085 & 44.608 & 39.607 & 43.725 & 38.569 \\
\hline CenterSpeller & VPibh & 18.407 & 11.128 & 16.535 & 16.797 & 15.199 & 19.050 \\
\hline CenterSpeller & VPibi & 28.770 & 31.514 & 31.356 & 27.147 & 27.185 & 26.935 \\
\hline CenterSpeller & VPibj & 22.652 & 24.898 & 24.808 & 18.391 & 16.365 & 18.844 \\
\hline CenterSpeller & VPica & 25.320 & 24.957 & 27.354 & 25.990 & 24.959 & 25.704 \\
\hline CenterSpeller & VPsaf & 30.342 & 29.324 & 31.062 & 28.999 & 28.167 & 31.544 \\
\hline AMUSE & VPfar & 5.549 & 5.370 & 6.552 & 8.610 & 9.986 & 8.314 \\
\hline AMUSE & VPfau & 14.682 & 16.384 & 17.185 & 14.834 & 16.699 & 16.139 \\
\hline AMUSE & VPfav & 15.535 & 14.670 & 15.456 & 19.101 & 21.385 & 19.395 \\
\hline AMUSE & VPfaw & 8.541 & 12.963 & 12.811 & 14.112 & 16.804 & 16.256 \\
\hline AMUSE & VPfax & 10.425 & 8.435 & 10.154 & 11.186 & 10.287 & 9.579 \\
\hline AMUSE & VPfaz & 29.548 & 31.219 & 31.078 & 33.581 & 30.235 & 37.179 \\
\hline AMUSE & VPfca & 19.655 & 20.983 & 17.787 & 27.462 & 27.767 & 27.462 \\
\hline AMUSE & VPfcb & 7.303 & 8.242 & 10.630 & 8.356 & 10.413 & 11.195 \\
\hline
\end{tabular}


Table 15 continued

\begin{tabular}{|c|c|c|c|c|c|c|c|}
\hline Dataset & Subj. & $\begin{array}{l}\text { SBF } \\
\text { L1-SVM }\end{array}$ & $\begin{array}{l}\text { SBF } \\
\text { L2-SVM }\end{array}$ & $\begin{array}{l}\text { SBF } \\
\text { M-SVM }\end{array}$ & $\begin{array}{l}\text { OSBF } \\
\text { L1-SVM }\end{array}$ & $\begin{array}{l}\text { OSBF } \\
\text { L2-SVM }\end{array}$ & $\begin{array}{l}\text { OSBF } \\
\text { M-SVM }\end{array}$ \\
\hline AMUSE & VPfcc & 20.129 & 22.135 & 24.316 & 22.509 & 25.576 & 22.971 \\
\hline AMUSE & VPfcd & 15.004 & 14.126 & 13.374 & 13.763 & 15.183 & 14.970 \\
\hline AMUSE & VPfcg & 8.621 & 10.283 & 7.204 & 9.691 & 9.228 & 9.166 \\
\hline AMUSE & VPfch & 1.838 & 1.146 & 1.469 & 2.819 & 2.862 & 3.136 \\
\hline AMUSE & VPfcj & 3.197 & 2.200 & 3.654 & 4.565 & 5.378 & 7.027 \\
\hline AMUSE & VPfck & 9.682 & 9.056 & 9.368 & 9.594 & 10.557 & 10.408 \\
\hline AMUSE & VPfcm & 5.763 & 7.058 & 4.565 & 9.227 & 7.871 & 8.002 \\
\hline AMUSE & VPkw & 27.244 & 22.588 & 28.013 & 22.433 & 23.480 & 24.398 \\
\hline MVEP & VPfat & 14.958 & 14.291 & 13.818 & 17.155 & 15.372 & 14.972 \\
\hline MVEP & VPgdf & 5.504 & 5.419 & 3.910 & 5.314 & 5.845 & 6.016 \\
\hline MVEP & VPgdg & 6.586 & 11.509 & 9.982 & 8.776 & 8.822 & 9.048 \\
\hline MVEP & VPiac & 11.131 & 8.422 & 7.097 & 6.886 & 8.350 & 8.398 \\
\hline MVEP & VPiba & 4.186 & 5.435 & 7.175 & 5.528 & 4.035 & 6.015 \\
\hline MVEP & VPibe & 16.537 & 17.687 & 16.239 & 18.598 & 21.599 & 18.751 \\
\hline MVEP & VPibs & 8.185 & 6.961 & 6.306 & 6.972 & 9.618 & 9.155 \\
\hline MVEP & VPibt & 9.232 & 10.368 & 9.426 & 12.149 & 11.743 & 12.149 \\
\hline MVEP & VPibu & 2.253 & 3.136 & 2.854 & 4.108 & 3.430 & 3.672 \\
\hline MVEP & VPibv & 10.438 & 7.861 & 10.650 & 14.113 & 12.383 & 13.729 \\
\hline MVEP & VPibw & 13.403 & 13.192 & 17.706 & 17.485 & 19.418 & 15.786 \\
\hline MVEP & VPibx & 15.977 & 14.671 & 15.071 & 16.467 & 16.972 & 15.894 \\
\hline MVEP & VPiby & 10.564 & 7.123 & 9.098 & 6.992 & 8.197 & 8.767 \\
\hline MVEP & VPice & 5.280 & 4.897 & 5.019 & 5.710 & 6.264 & 6.892 \\
\hline MVEP & VPicv & 7.271 & 7.813 & 6.522 & 7.422 & 7.343 & 7.215 \\
\hline Akimpech & ACS & 23.354 & 29.594 & 27.111 & 22.840 & 23.990 & 24.666 \\
\hline Akimpech & APM & 51.115 & 53.921 & 54.563 & 50.551 & 51.691 & 48.586 \\
\hline Akimpech & ASG & 40.801 & 43.749 & 42.566 & 36.551 & 40.254 & 41.175 \\
\hline Akimpech & ASR & 22.004 & 20.972 & 13.311 & 22.561 & 20.642 & 16.259 \\
\hline Akimpech & CLL & 24.106 & 29.092 & 30.928 & 23.146 & 29.745 & 32.356 \\
\hline Akimpech & CLR & 52.884 & 47.413 & 45.833 & 45.833 & 41.666 & 39.285 \\
\hline Akimpech & DCM & 45.059 & 42.503 & 42.313 & 42.218 & 43.689 & 40.103 \\
\hline Akimpech & DLP & 21.249 & 31.112 & 28.765 & 28.278 & 28.668 & 25.819 \\
\hline Akimpech & DMA & 18.975 & 25.073 & 24.142 & 25.724 & 28.174 & 26.192 \\
\hline Akimpech & ELC & 53.088 & 50.737 & 53.710 & 50.182 & 52.083 & 48.076 \\
\hline Akimpech & FSZ & 34.140 & 45.379 & 29.082 & 32.874 & 35.871 & 34.417 \\
\hline Akimpech & GCE & 25.441 & 28.303 & 26.500 & 25.405 & 25.961 & 24.513 \\
\hline Akimpech & ICE & 35.864 & 38.841 & 28.723 & 35.256 & 34.203 & 33.536 \\
\hline Akimpech & $\mathrm{IZH}$ & 31.358 & 32.417 & 28.353 & 31.212 & 32.067 & 29.545 \\
\hline Akimpech & JCR & 17.051 & 21.163 & 22.774 & 23.031 & 23.047 & 19.216 \\
\hline
\end{tabular}


Table 15 continued

\begin{tabular}{llllllll}
\hline Dataset & Subj. & $\begin{array}{l}\text { SBF } \\
\text { L1-SVM }\end{array}$ & $\begin{array}{l}\text { SBF } \\
\text { L2-SVM }\end{array}$ & $\begin{array}{l}\text { SBF } \\
\text { M-SVM }\end{array}$ & $\begin{array}{l}\text { OSBF } \\
\text { L1-SVM }\end{array}$ & $\begin{array}{l}\text { OSBF } \\
\text { L2-SVM }\end{array}$ & $\begin{array}{l}\text { OSBF } \\
\text { M-SVM }\end{array}$ \\
\hline Akimpech & JLD & 49.818 & 45.833 & 39.010 & 44.788 & 47.577 & 42.177 \\
Akimpech & JMR & 29.005 & 41.494 & 39.660 & 37.878 & 41.044 & 39.746 \\
Akimpech & JSC & 17.758 & 27.944 & 25.540 & 19.469 & 28.301 & 25.784 \\
Akimpech & JST & 53.710 & 57.053 & 55.220 & 51.691 & 54.347 & 51.115 \\
Akimpech & LAC & 58.760 & 57.772 & 60.043 & 55.443 & 50.182 & 52.083 \\
Akimpech & LAG & 39.163 & 38.809 & 41.354 & 36.548 & 41.729 & 36.667 \\
Akimpech & LGP & 41.540 & 50.737 & 51.497 & 46.928 & 51.497 & 50.551 \\
Akimpech & LPS & 21.166 & 23.951 & 22.754 & 22.754 & 22.754 & 24.598 \\
Akimpech & MoMR & 36.569 & 37.083 & 33.674 & 35.714 & 32.850 & 35.166 \\
Akimpech & PGA & 17.631 & 24.322 & 21.851 & 22.807 & 24.922 & 22.353 \\
Akimpech & WFG & 31.372 & 41.167 & 37.770 & 34.324 & 33.587 & 35.640 \\
Akimpech & XCL & 41.036 & 52.083 & 46.840 & 42.640 & 45.833 & 40.279 \\
\hline
\end{tabular}

\section{References}

Bnci horizon website. http://bnci-horizon-2020.eu/database/data-sets

Aricò, P., Aloise, F., Schettini, F., Salinari, S., Mattia, D., \& Cincotti, F. (2014). Influence of P300 latency jitter on event related potential-based brain-computer interface performance. Journal of Neural Engineering, 11(3),

Bianchi, L. (2018). The npxlab suite 2018: A free features rich set of tools for the analysis of neuro-electric signals. WSEAS Transactions on Systems and Control, 13(3), 145-152.

Bianchi, L. (2020). A videogame driven by the mind: Are motor acts necessary to play? In: Advances in intelligent systems and computing, 2020, FICC, pp. 40-50

Bianchi, L., Liti, C., \& Piccialli, V. (2019). A new early stopping method for p300 spellers. IEEE Transactions on Neural Systems and Rehabilitation Engineering, 27(8), 1635-1643. https://doi.org/10.1109/TNSRE. 2019.2924080.

Bockbrader, M. A., Francisco, G., Lee, R., Olson, J., Solinsky, R., \& Boninger, M. L.: Brain computer interfaces in rehabilitation medicine. PM\&R 10(9S2), S233-S243 (2018). https://doi.org/10.1016/j.pmrj.2018.05. 028 .

Chaovalitwongse, W. A., Prokopyev, O. A., \& Pardalos, P. M. (2006). Electroencephalogram (eeg) time series classification: Applications in epilepsy. Annals of Operations Research, 148(1), 227-250. https://doi. org/10.1007/s10479-006-0076-x.

Draper, N. R., \& Smith, H. (1998). Applied regression analysis (Vol. 326). New York: Wiley.

Fan, R. E., Chang, K. W., Hsieh, C. J., Wang, X. R., \& Lin, C. J. (2008). Liblinear: A library for large linear classification. Journal of Machine Learning Research, 9, 1871-1874.

Farwell, L. A., \& Donchin, E. (1988). Talking off the top of your head: Toward a mental prosthesis utilizing event-related brain potentials. Electroencephalography and Clinical Neurophysiology, 70(6), 510-523.

Friedman, J., Hastie, T., \& Tibshirani, R. (2001) The elements of statistical learning, vol. 1. Springer series in statistics New York.

Gu, Z., Chen, Z., Zhang, J., Zhang, X., \& Yu, Z. L. (2019). An online interactive paradigm for P300 braincomputer interface speller. IEEE Transactions on Neural Systems and Rehabilitation Engineering, 27(2), 152-161.

Höhne, J., Schreuder, M., Blankertz, B., \& Tangermann, M.: Two-dimensional auditory P300 speller with predictive text system. In: 2010 Annual international conference of the IEEE engineering in medicine and biology, pp. 4185-4188 (2010). https://doi.org/10.1109/IEMBS.2010.5627379

Hsieh, C. J., Chang, K. W., Lin, C. J., Keerthi, S. S., \& Sundararajan, S. (2008) A dual coordinate descent method for large-scale linear svm. In: Proceedings of the 25th international conference on machine learning, pp. 408-415 
Huang, Y., He, F., Xu, M., \& Qi, H. (2020). Operate P300 speller when performing other task. Journal of Neural Engineering. http://doi.org/10.1088/1741-2552/abb4a6.

Jiang, J., Yin, E., Wang, C., Xu, M., \& Ming, D. (2018). Incorporation of dynamic stopping strategy into the high-speed SSVEP-based BCIs. Journal of Neural Engineering, 15(4), 046025.

Jin, J., Allison, B. Z., Sellers, E. W., Brunner, C., Horki, P., Wang, X., et al. (2011). An adaptive P300-based control system. Journal of Neural Engineering, 8(3), 036006.

Kha, V. A., Nguyen, D. N., Kha, H. H., \& Dutkiewicz, E. (2017). Dynamic stopping using eSVM scores analysis for event-related potential brain-computer interfaces. In: 2017 11th international symposium on medical information and communication technology (ISMICT), pp. 82-85.

Khojandi, A., Shylo, O., \& Zokaeinikoo, M. (2019). Automatic eeg classification: A path to smart and connected sleep interventions. Annals of Operations Research, 276(1), 169-190. https://doi.org/10.1007/s10479018-2823-1.

Krusienski, D. J., Sellers, E. W., Cabestaing, F., Bayoudh, S., McFarland, D. J., Vaughan, T. M., et al. (2006). A comparison of classification techniques for the P300 speller. Journal of Neural Engineering, 3(4), 299.

Krusienski, D. J., Sellers, E. W., McFarland, D. J., Vaughan, T. M., \& Wolpaw, J. R. (2008). Toward enhanced p300 speller performance. Journal of Neuroscience Methods, 167(1), 15-21.

Ledesma-Ramirez, C., Bojorges-Valdez, E., Yáñez-Suarez, O., Saavedra, C., Bougrain, L., \& Gentiletti, G. G. (2010). An open-access p300 speller database

Lenhardt, A., Kaper, M., \& Ritter, H. (2008). An adaptive P300-based online Brain' computer interface. IEEE Transactions on Neural Systems and Rehabilitation Engineering, 16(2), 121-130. https://doi.org/10. 1109/TNSRE.2007.912816.

Liu, T., Goldberg, L., Gao, S., \& Hong, B. (2010). An online brain-computer interface using non-flashing visual evoked potentials. Journal of Neural Engineering, 7(3), 036003.

Lotte, F.: A tutorial on eeg signal-processing techniques for mental-state recognition in brain-computer interfaces. In: Guide to brain-computer music interfacing, pp. 133-161. Springer (2014)

Lotte, F., Bougrain, L., Cichocki, A., Clerc, M., Congedo, M., Rakotomamonjy, A., et al. (2018). A review of classification algorithms for EEG-based brain-computer interfaces: A 10 year update. Journal of Neural Engineering, 15(3),

Lugo, Z. R., Quitadamo, L. R., Bianchi, L., Pellas, F., Veser, S., Lesenfants, D., Real, R. G. L., Herbert, C., Guger, C., Kotchoubey, B., Mattia, D., Kbler, A., Laureys, S., \& Noirhomme, Q. (2016). Cognitive processing in non-communicative patients: what can event-related potentials tell us? Frontiers in Human Neuroscience 10, 569. https://doi.org/10.3389/fnhum.2016.00569.

Mainsah, B. O., Colwell, K. A., Collins, L. M., \& Throckmorton, C. S. (2014). Utilizing a language model to improve online dynamic data collection in P300 spellers. IEEE Transactions on Neural Systems and Rehabilitation Engineering, 22(4), 837-846.

McCane, L.M., Heckman, S.M., McFarland, D.J., Townsend, G., Mak, J.N., Sellers, E.W., Zeitlin, D., Tenteromano, L.M., Wolpaw, J.R., Vaughan, T.M. (2015). P300-based brain-computer interface (bci) event-related potentials (erps): People with amyotrophic lateral sclerosis (als) vs. age-matched controls. Clinical Neurophysiology 126(11), 2124-2131

Muller-Putz, G. R., \& Pfurtscheller, G. (2008). Control of an Electrical Prosthesis With an SSVEP-Based BCI. IEEE Transactions on Biomedical Engineering, 55(1), 361-364. https://doi.org/10.1109/TBME.2007. 897815.

Piccialli, V., Sciandrone, M.: Nonlinear optimization and support vector machines. 4OR 16(2), 111-149(2018)

Poli, R., Valeriani, D., \& Cinel, C. (2014). Collaborative brain-computer interface for aiding decision-making. PloS One, 9(7), e102693.

Quitadamo, L. R., Marciani, M. G., Cardarilli, G. C., \& Bianchi, L. (2008). Describing different brain computer interface systems through a unique model: A UML implementation. Neuroinformatics, 6(2), 81-96.

Riccio, A., Simione, L., Schettini, F., Pizzimenti, A., Inghilleri, M., Olivetti Belardinelli, M., et al. (2013). Attention and P300-based BCI performance in people with amyotrophic lateral sclerosis. Frontiers in Human Neuroscience, 7, 732.

Schaeff, S., Treder, M. S., Venthur, B., \& Blankertz, B. (2012). Exploring motion veps for gaze-independent communication. Journal of Neural Engineering, 9(4), 045006.

Schreuder, M., Höhne, J., Blankertz, B., Haufe, S., Dickhaus, T., \& Tangermann, M. (2013). Optimizing event-related potential based brain-computer interfaces: A systematic evaluation of dynamic stopping methods. Journal of Neural Engineering, 10(3), 036025.

Schreuder, M., Rost, T., \& Tangermann, M. (2011). Listen, you are writing! speeding up online spelling with a dynamic auditory BCI. Frontiers in Neuroscience, $5,112$.

Sellers, E. W., Krusienski, D. J., McFarland, D. J., Vaughan, T. M., \& Wolpaw, J. R. (2006). A P300 eventrelated potential brain-computer interface $(\mathrm{BCI})$ : The effects of matrix size and inter stimulus interval on performance. Biological Psychology, 73(3), 242-252. 
Sellers, E. W., Kubler, A., \& Donchin, E. (2006). Brain-computer interface research at the university of south florida cognitive psychophysiology laboratory: The P300 Speller. IEEE Transactions on Neural Systems and Rehabilitation Engineering, 14(2), 221-224.

Shahriari, Y., Vaughan, T. M., McCane, L., Allison, B. Z., Wolpaw, J. R., \& Krusienski, D. J. (2019). An exploration of BCI performance variations in people with amyotrophic lateral sclerosis using longitudinal EEG data. Journal of Neural Engineering, 16(5), 056031.

Sur, S., \& Sinha, V. (2009). Event-related potential: An overview. Industrial Psychiatry Journal, 18(1), 70.

Throckmorton, C. S., Colwell, K. A., Ryan, D. B., Sellers, E. W., \& Collins, L. M. (2013). Bayesian approach to dynamically controlling data collection in P300 spellers. IEEE Transactions on Neural Systems and Rehabilitation Engineering, 21(3), 508-517.

Treder, M. S., \& Blankertz, B. (2010). (c) overt attention and visual speller design in an erp-based braincomputer interface. Behavioral and Brain Functions, 6(1), 28.

Treder, M. S., Schmidt, N. M., \& Blankertz, B. (2011). Gaze-independent brain-computer interfaces based on covert attention and feature attention. Journal of Neural Engineering, 8(6), 066003.

Valeriani, D., \& Poli, R. (2019). Cyborg groups enhance face recognition in crowded environments. PloS One, 14(3), e0212935.

Vo, K., Nguyen, D. N., Kha, H. H., \& Dutkiewicz, E. (2017). Subject-independent P300 BCI using ensemble classifier, dynamic stopping and adaptive learning. In: GLOBECOM 2017-2017 IEEE global communications conference, pp. 1-7

Vo, K., Pham, T., Nguyen, D. N., Kha, H. H., \& Dutkiewicz, E. (2018). Subject-independent ERP-based brain-computer interfaces. IEEE Transactions on Neural Systems and Rehabilitation Engineering, 26(4), 719-728. https://doi.org/10.1109/tnsre.2018.2810332.

Wolpaw, J., \& Wolpaw, E. W. (2012). Brain-computer interfaces: Principles and practice. New York: Oxford University Press.

Wolpaw, J. R., Ramoser, H., McFarland, D. J., \& Pfurtscheller, G. (1998). EEG-based communication: Improved accuracy by response verification. IEEE Transactions on Rehabilitation Engineering, 6(3), 326-333.

Zhang, H., Guan, C., \& Wang, C. (2008). Asynchronous P300-based brain-computer interfaces: A computational approach with statistical models. IEEE Transactions on Biomedical Engineering, 55(6), 1754-1763.

Publisher's Note Springer Nature remains neutral with regard to jurisdictional claims in published maps and institutional affiliations.

\section{Affiliations}

\section{Luigi Bianchi ${ }^{1} \cdot$ Chiara Liti $^{1} \cdot$ Giampaolo Liuzzi $^{2} \cdot$ Veronica Piccialli $^{1}$ (D) Cecilia Salvatore ${ }^{1}$}

$\bowtie$ Veronica Piccialli

veronica.piccialli@uniroma2.it

Luigi Bianchi

luigi.bianchi@uniroma2.it

Chiara Liti

chiara.liti@uniroma2.it

Giampaolo Liuzzi

giampaolo.liuzzi@diag.uniroma1.it

Cecilia Salvatore

cecilia.salvatore@uniroma2.it

1 Department of Civil Engineering and Computer Science, University of Rome Tor Vergata, Rome, Italy

2 Department of Computer, Control and Management Engineering, Sapienza University of Rome, Rome, Italy 